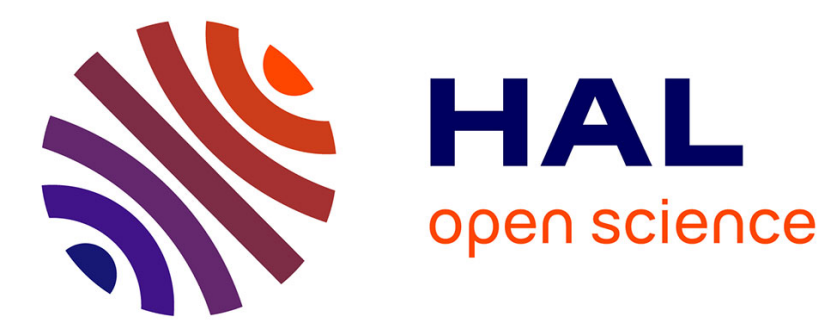

\title{
Goal-oriented updating of mechanical models using the adjoint framework
}

Ludovic Chamoin, Pierre Ladevèze, Julien Waeytens

\section{To cite this version:}

Ludovic Chamoin, Pierre Ladevèze, Julien Waeytens. Goal-oriented updating of mechanical models using the adjoint framework. Computational Mechanics, 2014, 54 (6), pp.1415-1430. 10.1007/s00466014-1066-5 . hal-01057077

\section{HAL Id: hal-01057077 https://hal.science/hal-01057077}

Submitted on 21 Aug 2014

HAL is a multi-disciplinary open access archive for the deposit and dissemination of scientific research documents, whether they are published or not. The documents may come from teaching and research institutions in France or abroad, or from public or private research centers.
L'archive ouverte pluridisciplinaire HAL, est destinée au dépôt et à la diffusion de documents scientifiques de niveau recherche, publiés ou non, émanant des établissements d'enseignement et de recherche français ou étrangers, des laboratoires publics ou privés. 


\title{
Goal-oriented updating of mechanical models using the adjoint framework
}

\author{
Ludovic Chamoin • Pierre Ladevèze • Julien Waeytens
}

Received : date / Accepted : date

\begin{abstract}
In this paper, we introduce a goal-oriented procedure for the updating of mechanical models. It is based as usual on information coming from measurement data, but these data are post-processed in a convenient way in order to firstly update model parameters which are the most influent for the prediction of a given quantity of interest. The objective is thus to perform a partial model calibration that enables to obtain an approximate value of the quantity of interest with sufficient accuracy and minimal model identification effort. The updating method uses the constitutive relation error framework as well as duality and adjoint techniques. It leads to a convenient strategy, mainly based on sensitivity analysis, that selects the relevant parameter set to be updated and also provides for useful quantitative tools in order to define optimal experiments. Performances of the approach are analyzed on examples involving linear elasticity and transient thermal models.
\end{abstract}

Keywords Model updating - Quantity of interest . Constitutive relation error · Sensitivity analysis

Mathematics Subject Classification (2000) 74G75. 74B05 $\cdot 74 \mathrm{~S} 05$

\section{Introduction}

Mathematical models are fundamental in science and engineering activities, particularly due to the fact that

L. Chamoin · P. Ladevèze

LMT-Cachan (ENS Cachan / CNRS / Paris 6 University),

61 Avenue du Président Wilson, 94235 Cachan Cedex, France

E-mail: $\{$ chamoin,ladeveze $\} @ l m t . e n s-c a c h a n . f r$

J. Waeytens

Paris-Est University, IFSTTAR,

14-20 Bd Newton 77447 Marne-La-Vallée Cedex 2, France

E-mail: julien.waeytens@ifsttar.fr they are the basic ingredient of numerical simulations that enable to reproduce physical phenomena and make predictions. However, a major concern is the capability of these models to represent a faithful abstraction of the real world. To address this issue and control the error between physical and mathematical models, model validation methods have been used for a long time $[34,33$, 32]. In such methods, model parameters are identified or updated in order to minimize the discrepancy between numerical predictions and experimental measurements. The process leads to inverse problems [8] which are usually ill-posed and require special care and specific techniques, such as regularization techniques proposed in [38], in order to ensure solvability. Getting missing information of the model from measurements is a procedure that is now commonly used in many scientific fields such as geophysics where soil characteristics (density, permeability) are studied, non-destructive testing to identify defects [3], or imaging where we can obtain images from a noised version using deconvolution [9] or detect damaged tissues using tomography $[4,16]$.

We focus here on Computational Mechanics models, in which a major component is the constitutive equation that describes the local behavior of the material. It is characterized by a set of material parameters whose values may highly influence results given by numerical simulations. In order to reduce modeling errors, it is thus important to address the issue of constitutive models calibration. For complex models, it is difficult to design experiments where observed model responses depend explicitly on model parameters. We rather use in practice experimental data that depend implicitly on these parameters, and the calibration is then viewed as an optimization problem in which parameter values are searched such that optimal agreement between experi- 
mental and simulated responses is achieved [12].

When a large number of experimental data is available, as it is the case with full-field kinematic measurements performed by means of imaging techniques (DIC for instance $[22,36]$ ), many calibrations methods can be applied for inverse analysis and identification of material properties [5]. Among all of them, we can mention the Equilibrium Gap Method (EGM) which is based on the discretization of equilibrium equations and minimization of equilibrium residuals, the FEMU method (balance method FEMU-F or displacement method FEM $\mathrm{U})$ which is an intuitive approach that consists in performing iteratively finite element computations to find constitutive parameters that achieve the best match between computed and actual measurements, or the Constitutive Equation Gap Method (CEGM) which was initially developed for updating finite element models from vibrational data and assessing quality of finite element meshes.

We consider in this paper the case where only few localized measurements are available. Again, several procedures exist in this framework to identify parameters, such as minimization of cost functions associated with regularization techniques [8], or the Bayesian inference approach that formulates parameter identification in a stochastic setting $[37,35]$. Using the concept of Constitutive Relation Error (CRE) defines another model calibration method on which we focus here. First introduced in $[26,7,13,27,14]$ for dynamics models, this method was latter successfully used in many calibration applications including defects [11], uncertain measurements and behaviors [29,17], or corrupted measurements [1]. Recent applications of the method dealt with the updating of models used in bolted assemblies [19], or association with PGD reduced models to deal with realtime calibration in machining processes [10]. The use of the CRE presents interesting advantages; it has excellent capacities to localize structural defects spatially, it is very robust with respect to noisy measurements, and it has good convexity properties. In this method, reliable theoretical and experimental information (equilibrium, sensor position) are favored compared to other information (material behavior, sensor measures). Furthermore, the employed hierarchical updating (only most erroneous zones are corrected) is a strategy that directly leads to a regularization process, required for ill-posed problems.

In this paper, we deal with mechanical models on large structures which are used both for the model calibration procedure and for prediction using numerical simulation. We consider that the prediction target is only the value of a given output of the model, denoted quantity of interest in the following, that implicitly depends on model parameters. Therefore, if the quantity of interest is not very sensitive with respect to some parameters, there is probably no need to estimate these parameters with high accuracy. This goal-oriented model updating approach is motivated by the fact that the objective of numerical simulations is usually not the global response of the model, but only specific features which are relevant for design (such as local stress, maximal displacement or temperature, etc.). It aims at - performing a partial calibration of the model so as to ensure the quality of predicted quantities of interest with a minimal calibration effort.

Several works have already addressed this scientific issue. In $[6,24]$, an optimization problem coupled with a dual method was introduced to assess, in a goal quantity, the sensitivity with respect to the observed data (uncertainty or noise), as well as discretization error affecting the computed value of parameters. The a posteriori discretization error estimator was also used in an adaptive algorithm to construct economic meshes. In [25], the goal-oriented a posteriori error estimation for identification problems was extended to accommodate the combined identification and subsequent simulation problems which may be governed by different state equations (and only coupled via model parameters). For a given tolerance in a quantity of interest, depending on the solution of the simulation problem, three sources of errors were controlled: modeling error, discretization error polluting the identification problem, and discretization error in the simulation.

Here, we wish to go one step further and define a goaloriented version of updating methods performed using the CRE. We assume that the discretization error is negligible compared to the modeling error, and focus on the sensitivity of the considered quantity of interest with respect to parameters and measurements. On the one hand, we introduce new dedicated cost functions that lead to a convenient goal-oriented updating process, selecting automatically the relevant model parameters that need to be updated for the prediction of the quantity of interest. On the other hand, we define quantitative sensitivity tools that enable to set up optimal experiments and measurements (sensor location, type of measure) with respect to the output of interest to predict. For the sake of simplicity, we present the new method and tools in the framework of linear elasticity models, whereas numerical experiments will also involve time-dependent problems.

The paper is organized as follows: after this introduction, Section 2 presents the reference mathematical 
model which is considered throughout the paper as well as the classical setting for model calibration based on the minimization of cost functions; Section 3 focuses on model calibration performed using the concept of CRE; Section 4 introduces the goal-oriented framework that enables to update a model, still using the CRE, but with respect to a quantity of interest; a sensitivity analysis is presented in Section 5 in order to set up an optimal experimental procedure for the goal-oriented calibration; eventually, numerical experiments are conducted in Section 6, before drawing concluding remarks and prospects in Section 7.

\section{Mathematical model and general updating strategy}

\subsection{The reference mathematical model}

We consider a linear elastic body whose undeformed configuration occupies domain $\Omega \subset \mathbb{R}^{d}$, with boundary $\partial \Omega$ (Figure 1). It is subjected to a given displacement field $\mathbf{u}_{d}$ on $\partial_{1} \Omega \subset \partial \Omega$, whereas a body force field $\mathbf{f}_{d}$ and a traction force field $\mathbf{F}_{d}$ are imposed in $\Omega$ and on $\partial_{2} \Omega$, respectively, with $\partial_{1} \Omega \cup \partial_{2} \Omega=\partial \Omega$ and $\partial_{1} \Omega \cap$ $\partial_{2} \Omega=\emptyset$. The corresponding model is governed by three sets of equations, namely the kinematic compatibility equations:

$\mathscr{E}(\mathbf{u})=\frac{1}{2}\left(\nabla \mathbf{u}+\nabla^{T} \mathbf{u}\right) \quad$ in $\Omega \quad ; \quad \mathbf{u}=\mathbf{u}_{d} \quad$ on $\partial_{1} \Omega$

the equilibrium equations:

$\operatorname{div} \sigma+\mathbf{f}_{d}=\mathbf{0} \quad$ in $\Omega \quad ; \quad \sigma \mathbf{n}=\mathbf{F}_{d} \quad$ on $\partial_{2} \Omega$

and the constitutive equation:

$\sigma=\boldsymbol{K}_{\mathbb{E}}(\mathbf{u})$ in $\Omega$

$\mathbf{u}$ is the displacement vector, $\notin$ the linearized strain tensor, $\sigma$ the Cauchy stress tensor, and $\mathbf{n}$ the outward unit normal vector. $\boldsymbol{K}$ denotes the Hooke tensor, possibly heterogeneous, and we suppose it is described by a set $\mathbf{p}$ of $n_{p}$ scalar parameters that belong to a bounded space $\mathcal{P} \subset \mathbb{R}^{n_{p}}$. For instance, if the elastic properties are isotropic, $\boldsymbol{K}$ is described (at each point) in terms of two independent scalar moduli.

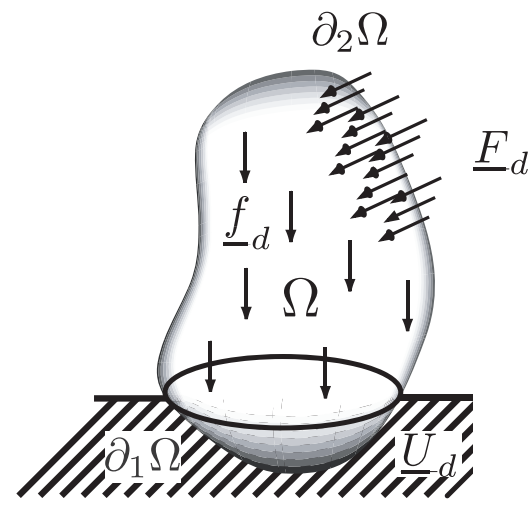

Fig. 1 The structure and its environment.

We introduce classical sets $\mathcal{U}$ and $\mathcal{S}$ of kinematically admissible displacement fields and statically admissible stress fields, respectively. $\mathcal{U}$ is defined as the set of vector fields $\mathbf{v} \in\left[H^{1}(\Omega)\right]^{d}$ verifying (1), whereas $\mathcal{S}$ is defined as the set of symmetric second-order tensor fields $\pi \in\left[L^{2}(\Omega)\right]^{d(d+1) / 2}$ verifying (2).

Equations (1-3) constitute a direct problem that can be recast in the following weak form:

Find $\mathbf{u}(\mathbf{p}) \in \mathcal{U}$ such that

$\mathcal{A}(\mathbf{p}, \mathbf{u}, \mathbf{v})=a(\mathbf{p}, \mathbf{u}, \mathbf{v})-l(\mathbf{v})=0 \quad \forall \mathbf{v} \in \mathcal{U}_{0}$

where $\mathcal{U}_{0}$ denotes the vectorial space associated with $\mathcal{U}$, and forms $a$ and $l$ are defined as:

$$
\begin{aligned}
a(\mathbf{p}, \mathbf{u}, \mathbf{v}) & =\int_{\Omega} \boldsymbol{K}(\mathbf{p}) \mathscr{E}(\mathbf{u}): \mathbb{E}(\mathbf{v}) \mathrm{d} \Omega \\
l(\mathbf{v}) & =\int_{\Omega} \mathbf{f}_{d} \cdot \mathbf{v} \mathrm{d} \Omega+\int_{\partial_{2} \Omega} \mathbf{F}_{d} \cdot \mathbf{v} \mathrm{d} S
\end{aligned}
$$

This problem is well-posed in the Hadamard sense i.e. the solution exists, is unique and stable with respect to data.

\subsection{General updating strategy}

We assume in the following that the value of $\mathbf{p}$ is not known and needs to be updated using additional information i.e. given experimental data. These data are represented by a set of observable quantities $\mathbf{s}_{\text {obs }}$ measured from the response of the body on selected points $\mathbf{x}_{i}\left(i=1, \ldots, n_{o b s}\right)$ located outside $\partial_{1} \Omega$. The updating process then requires to solve an inverse problem which is usually ill-posed.

The inversion technique is often formulated as a minimization of a cost function $\mathcal{F}$, so as to ensure existence 
of a solution $\mathbf{p}_{\text {sol }}$ in $\mathcal{P}$ :

$\mathbf{p}_{\text {sol }}=\underset{\mathbf{p} \in \mathcal{P}}{\operatorname{argmin}} \mathcal{F}(\mathbf{p})$

Remark 1 As regards uniqueness of $\mathbf{p}_{\text {sol }}$, considering a strictly convex cost function $\mathcal{F}$ is a sufficient condition.

Generally, a least-square minimization is used i.e. $\mathcal{F}$ is constructed by means of the Euclidean norm involving solution $\mathbf{u}(\mathbf{p})$ and experimental data $\mathbf{s}_{\text {obs }}$ :

$$
\begin{aligned}
\mathcal{F}(\mathbf{p}, \mathbf{u}(\mathbf{p})) & =\frac{1}{2}\left\|\mathbf{s}[\mathbf{u}(\mathbf{p})]-\mathbf{s}_{o b s}\right\|^{2} \\
& =\frac{1}{2} \sum_{i=1}^{n_{o b s}} c_{i}\left\|\mathbf{s}[\mathbf{u}(\mathbf{p})]\left(\mathbf{x}_{i}\right)-\mathbf{s}_{o b s}\left(\mathbf{x}_{i}\right)\right\|^{2}
\end{aligned}
$$

s being the extraction operator associated with observations, and $c_{i}$ some scalar parameters. The minimization, under the constraint that $\mathbf{u}$ satisfies state equation (4), is usually performed by means of first order steepest descent methods. In this context, a convenient and widely used method to evaluate the gradient $\nabla_{\mathbf{p}} \mathcal{F}$ is the adjoint state method; introducing the Lagrangian $\mathcal{L}(\mathbf{p}, \mathbf{u}, \boldsymbol{\lambda})=\mathcal{F}(\mathbf{p}, \mathbf{u})-\mathcal{A}(\mathbf{p}, \mathbf{u}, \boldsymbol{\lambda})$ and searching for its stationary point $\mathbf{z} \equiv(\mathbf{p}, \mathbf{u}, \boldsymbol{\lambda})$, it leads to the system:

$$
\begin{aligned}
& \mathcal{L}_{\mathbf{p}}^{\prime}(\mathbf{z} ; \delta \mathbf{p})=\mathcal{F}_{\mathbf{p}}^{\prime}(\mathbf{p}, \mathbf{u} ; \delta \mathbf{p})-\mathcal{A}_{\mathbf{p}}^{\prime}(\mathbf{p}, \mathbf{u}, \boldsymbol{\lambda} ; \delta \mathbf{p})=0 \\
& \mathcal{L}_{\mathbf{u}}^{\prime}(\mathbf{z} ; \delta \mathbf{u})=\mathcal{F}_{\mathbf{u}}^{\prime}(\mathbf{p}, \mathbf{u} ; \delta \mathbf{u})-\mathcal{A}(\mathbf{p}, \delta \mathbf{u}, \boldsymbol{\lambda})=0 \\
& \mathcal{L}_{\boldsymbol{\lambda}}^{\prime}(\mathbf{z} ; \delta \boldsymbol{\lambda})=\mathcal{A}(\mathbf{p}, \mathbf{u}, \delta \boldsymbol{\lambda})=0
\end{aligned}
$$

for all $(\delta \mathbf{p}, \delta \mathbf{u}, \delta \boldsymbol{\lambda}) \in \mathcal{P}_{0} \times \mathcal{U}_{0} \times \mathcal{U}_{0}$.

The second equation is the co-state equation also called adjoint equation. For a couple $(\mathbf{u}, \mathbf{p})$ that verifies the state equation (4), we get $\mathcal{L}(\mathbf{p}, \mathbf{u},, \boldsymbol{\lambda})=\mathcal{F}(\mathbf{p}, \mathbf{u})$. Furthermore, if $\mathbf{z}$ satisfies state and co-state equations, then:

$\mathrm{d}_{\mathbf{p}} \mathcal{F}(\mathbf{p}, \mathbf{u}(\mathbf{p}) ; \delta \mathbf{p})=\mathcal{L}_{\mathbf{p}}^{\prime}(\mathbf{z} ; \delta \mathbf{p})$

A priori information on $\mathbf{p}$ (bounds on parameter values, regularity conditions) can be inserted in the formulation to stabilize the inversion process and reduce sensitivity; these are regularization methods. A wellknown regularization method is the one of Tikhonov [38] in which the cost function takes the form:

$\mathcal{G}(\mathbf{p})=\mathcal{F}(\mathbf{p})+\sum_{j} \alpha_{j} R_{j}\left(p_{j}\right)$

where $\alpha_{j}$ are scalar regularization parameters and $R_{j}$ is a penalization functional for parameter $p_{j}$. Classical examples are $L^{2}$ penalization functional $R_{j}\left(p_{j}\right)=$ $\frac{1}{2}\left|p_{j}-p_{j}^{0}\right|^{2}$ and $H^{1}$ penalization functional $R_{j}\left(p_{j}\right)=$ $\frac{1}{2}\left\|\nabla p_{j}\right\|_{L^{2}}^{2}$.

\section{Calibration method based on the constitutive} relation error

\subsection{Basic ideas}

The concept of constitutive relation error (CRE), which has strong mechanical foundations, has been used for decades for a posteriori discretization error control in finite element computations [28]. It defines an energy measure, denoted $\mathcal{E}$, of the distance between a given stress field $\pi$ and another stress field obtained from a given displacement field $\mathbf{v}$ using (3):

$$
\begin{aligned}
& \mathcal{E}^{2}(\mathbf{p}, \mathbf{v}, \pi) \\
& =\frac{1}{2} \int_{\Omega}[\pi-\boldsymbol{K}(\mathbf{p}) \notin(\mathbf{v})]: \boldsymbol{K}^{-1}(\mathbf{p})[\pi-\boldsymbol{K}(\mathbf{p}) \notin(\mathbf{v})] \mathrm{d} \Omega
\end{aligned}
$$

Remark 2 A more general definition of $\mathcal{E}$, in particular for nonlinear constitutive models, can be set up using convex potentials associated with standard generalized materials [20].

Its usefulness is explained by the fact that the solution $(\mathbf{u}, \sigma)$ of the well-posed direct problem $(1-3)$ is characterized by:

$(\mathbf{u}, \sigma)=\underset{(\mathbf{v}, \pi) \in \mathcal{U} \times \mathcal{S}}{\operatorname{argmin}} \mathcal{E}(\mathbf{p}, \mathbf{v}, \pi) \quad ; \quad \mathcal{E}(\mathbf{p}, \mathbf{u}, \sigma)=0$

Furthermore, for any $(\mathbf{v}, \pi) \in \mathcal{U} \times \mathcal{S}$, the measure $\mathcal{E}(\mathbf{p}, \mathbf{v}, \pi)$ can be split into two terms:

$\mathcal{E}^{2}(\mathbf{p}, \mathbf{v}, \pi)=W_{p}(\mathbf{p}, \mathbf{v})+W_{c}(\mathbf{p}, \pi)$

where $W_{p}$ and $W_{c}$ are potential and complementary energy, respectively.

In model calibration formulations using the CRE, the cost function $\mathcal{F}$ is constructed from $\mathcal{E}$; it was shown that this choice presents convenient properties for inversion [18]. In first applications, experimental data used to be involved as boundary conditions in admissibility spaces, defining new spaces $\overline{\mathcal{U}}$ and $\overline{\mathcal{S}}$ and leading (except for special cases) to:

$\min _{(\mathbf{v}, \pi) \in \overline{\mathcal{U}} \times \overline{\mathcal{S}}} \mathcal{E}(\mathbf{p}, \mathbf{v}, \pi) \neq 0$

even though the solution realizing the minimum of $\mathcal{E}(\mathbf{p}, \mathbf{v}, \pi)$ over $\overline{\mathcal{U}} \times \overline{\mathcal{S}}$ is unique (to a given displacement of rigid body belonging to $\overline{\mathcal{U}}_{0}$ ) and a decomposition similar to (13) is still possible.

The inverse problem was thus defined as:

$\mathbf{p}_{\text {sol }}=\underset{\mathbf{p} \in \mathcal{P}}{\operatorname{argmin}} \mathcal{F}(\mathbf{p}) \quad ; \quad \mathcal{F}(\mathbf{p})=\min _{(\mathbf{v}, \pi) \in \overline{\mathcal{U}} \times \overline{\mathcal{S}}} \mathcal{E}^{2}(\mathbf{p}, \mathbf{v}, \pi)$ 
However, problem (15) is not well-posed in general and requires additional information on $\boldsymbol{K}(\mathbf{p})$, in particular for noisy measurements.

In more recent applications, boundary conditions coming from experimental data have been relaxed; they are not involved in admissibility spaces any more (we thus keep $\mathcal{U}$ and $\mathcal{S}$ ) but are imposed by penalization in a new definition of the constitutive relation error called modified constitutive relation error $[26,7,13]$ and denoted $\mathcal{E}_{m}$ :

$\mathcal{E}_{m}^{2}(\mathbf{p}, \mathbf{v}, \pi)=\mathcal{E}^{2}(\mathbf{p}, \mathbf{v}, \pi)+\frac{1}{2} \frac{r}{1-r}\left\|\mathbf{s}[\mathbf{v}]-\mathbf{s}_{o b s}\right\|_{L^{2}}^{2}$

The two terms composing $\mathcal{E}_{m}$ can be respectively seen as measures of modeling error and measurement error. $r$ is a scalar parameter that enables to modulate the influence of these terms. The philosophy of the modified constitutive relation error is thus to favor reliable theoretical and experimental information (equilibrium, sensor location) compared to other information (constitutive relation, sensor measurement).

Remark 3 The choice of $r$ should generally be made in regards of the a priori reliability on both model and measurements; for instance, Morozov principle or LCurve method [21] may be used to define $r$ with respect to the data noise. However, the influence of $r$ on the quality of the updating performed using CRE was deeply analyzed in several works, particularly in [15]. It was shown that the value $r=0.5$ gave the best insensitivity of the cost function with respect to measurement uncertainties. Consequently, we will choose this value throughout the paper.

The new inverse problem is defined as:

$\mathbf{p}_{\text {sol }}=\underset{\mathbf{p} \in \mathcal{P}}{\operatorname{argmin}} \mathcal{F}(\mathbf{p}) \quad ; \quad \mathcal{F}(\mathbf{p})=\min _{(\mathbf{v}, \pi) \in \mathcal{U} \times \mathcal{S}} \mathcal{E}_{m}^{2}(\mathbf{p}, \mathbf{v}, \pi)$

It leads to an iterative method, each iteration consisting of two partial minimizations steps which are detailed in the next section.

\subsection{Technical details on the minimization strategy}

As we do not consider discretization error in this paper, we explain the minimization strategy associated with (17) directly from discretized equations. The discretized modified CRE, denoted $\mathcal{E}_{m}^{h}$, reads:

$$
\begin{aligned}
\mathcal{E}_{m}^{h 2}(\mathbf{p}, \mathbf{U}, \mathbf{V}) & =\frac{1}{2}(\mathbf{U}-\mathbf{V})^{T} \mathbb{K}(\mathbf{p})(\mathbf{U}-\mathbf{V}) \\
& +\frac{1}{2} \frac{r}{1-r}\left(\mathbf{U}-\mathbf{U}_{o b s}\right)^{T} \mathbb{G}_{u}\left(\mathbf{U}-\mathbf{U}_{o b s}\right)
\end{aligned}
$$

$\mathbf{U} \in \mathcal{U}^{h}$ is kinematically admissible in the sense that it verifies discretized kinematic equations, whereas $\mathbf{V} \in$ $\mathcal{U}_{\mathcal{S}}^{h}$ is statically admissible in the sense that it verifies equilibrium equations in a finite element sense $\mathbb{K}(\mathbf{p}) \mathbf{V}=$ $\mathbf{F}$, with $\mathbb{K}(\mathbf{p})$ the stiffness matrix of the complete discretized structure. Consequently, $\mathbf{U}$ contains prescribed dofs contrary to $\mathbf{V}$. $\mathbb{G}_{u}$ is a scaling matrix which is in practice chosen so that both terms of $\mathcal{E}_{m}^{h}$ can be compared.

The first minimization step involved in (17), i.e. the computation of $\mathcal{F}^{h}(\mathbf{p})$ such that:

$\mathcal{F}^{h}(\mathbf{p})=\min _{(\mathbf{U}, \mathbf{V}) \in \mathcal{U}^{h} \times \mathcal{U}_{\mathcal{S}}^{h}} \mathcal{E}_{m}^{h 2}(\mathbf{p}, \mathbf{U}, \mathbf{V})$

is called the localization step. This is a constrained minimization problem, and we thus introduce the Lagrangian:

$$
L(\mathbf{U}, \mathbf{V}, \boldsymbol{\Lambda})=\mathcal{E}_{m}^{h 2}(\mathbf{p}, \mathbf{U}, \mathbf{V})-\boldsymbol{\Lambda}^{T}(\mathbb{K}(\mathbf{p}) \mathbf{V}-\mathbf{F})
$$

Finding its stationary point leads to the system:

$$
\begin{array}{r}
\widetilde{\mathbb{K}}(\mathbf{p})(\mathbf{U}-\mathbf{V})+\frac{r}{1-r} \widetilde{\mathbb{G}}_{u}\left(\mathbf{U}-\mathbf{U}_{o b s}\right)=\mathbf{0} \\
\mathbb{K}(\mathbf{p})(\mathbf{U}-\mathbf{V})+\mathbb{K}(\mathbf{p}) \boldsymbol{\Lambda}=\mathbf{0} \\
\mathbb{K}(\mathbf{p}) \mathbf{V}-\mathbf{F}=\mathbf{0}
\end{array}
$$

so that we obtain:

$$
\begin{aligned}
& \left(\widetilde{\mathbb{K}}(\mathbf{p})+\frac{r}{1-r} \widetilde{\mathbb{G}}_{u}\right) \mathbf{U}=\frac{r}{1-r} \widetilde{\mathbb{G}}_{u} \mathbf{U}_{o b s}+\widetilde{\mathbf{F}} \\
& \Longleftrightarrow \overline{\mathbb{K}} \mathbf{U}=\overline{\mathbf{F}}
\end{aligned}
$$

and can compute $\mathcal{F}^{h}(\mathbf{p})$. Quantities $\widetilde{\mathbb{K}}, \widetilde{\mathbb{G}}_{u}$ and $\widetilde{\mathbf{F}}$ are restrictions of $\mathbb{K}, \mathbb{G}_{u}$ and $\mathbf{F}$ in which lines corresponding to prescribed dofs for $\mathbf{U}$ have been removed.

Spatially slitting the modeling error term of $\mathcal{F}^{h}(\mathbf{p})$ into contributions of each element $p_{i}$ of $\mathbf{p}$ enables to localize and select the set of parameters that contribute the most to the error. Moreover, contributions of the measurement error enable to detect erroneous sensors.

The second minimization step involved in (17), i.e. the computation of $\mathbf{p}_{\text {sol }}$ such that:

$\mathbf{p}_{\text {sol }}=\underset{\mathbf{p} \in \mathcal{P}}{\operatorname{argmin}} \mathcal{F}^{h}(\mathbf{p})$ 
is called the correction step. In practice, $\mathcal{F}^{h}(\mathbf{p})$ is minimized with respect to parameters $p_{i}$ chosen in the previous step. This is a nonlinear process that uses an optimization algorithm, such as the gradient method with optimal path. This last method iterates such that $\mathbf{p}^{(k+1)}=\mathbf{p}^{(k)}+\alpha^{(k)} \mathbf{g}^{(k)} ; \mathbf{g}^{(k)}$ is chosen equal to $-\nabla \mathcal{F}^{h}\left(\mathbf{p}^{(k)}\right.$ and $\alpha^{(k)}$ corresponds to the minimum of $\mathcal{F}^{h}\left(\mathbf{p}^{(k)}+\right.$ $\left.x \mathbf{g}^{(k)}\right)$ for $x>0$.

The gradient of $\mathcal{F}^{h}(\mathbf{p})$ with respect to $p_{i}$ can be computed explicitly with low computational effort. Indeed, for the couple ( $\mathbf{U}, \mathbf{V})$ solution of the constrained minimization problem (19) and with $\boldsymbol{\Lambda}=\mathbf{V}-\mathbf{U}$, we have:

$$
\begin{aligned}
\mathrm{d}_{\mathbf{p}} \mathcal{F}^{h}(\mathbf{p} ; \delta \mathbf{p}) & =\mathcal{L}_{\mathbf{p}}^{\prime}(\mathbf{p}, \mathbf{U}, \mathbf{V}, \boldsymbol{\Lambda} ; \delta \mathbf{p}) \\
& =\frac{1}{2}(\mathbf{U}-\mathbf{V})^{T} \mathrm{~d}_{\mathbf{p}} \mathbb{K}(\mathbf{p} ; \delta \mathbf{p})(\mathbf{U}+\mathbf{V})
\end{aligned}
$$

Remark 4 As expected, we observe that the gradient vanishes when $\mathbf{U}=\mathbf{V}$.

The iterative process with localization and correction steps is in practice stopped when the cost function $\mathcal{F}^{h}(\mathbf{p})$ reaches a given tolerance value, without waiting for convergence. Furthermore, only parameters which are selected in the localization step are updated during the correction step (hierarchical updating). This naturally regularizes the inverse formulation.

\section{Goal-oriented updating with the constitutive relation error}

In the previous section, the model was updated globally with respect to experiments as a global (modified) constitutive relation error, defined on the whole domain $\Omega$ and with the whole parameter set $\mathbf{p}$, was used as a cost function. Furthermore, parameters to be updated first were selected using contributions of this global error.

Here, we aim at modifying the formulation to obtain an updating process which is dedicated to the prediction of given outputs of the model. Consequently, the identified set $\mathbf{p}$ is not expected to accurately model the actual structure; it is rather used to obtain an improved evaluation of outputs of interest from the a priori knowledge of the model and available data. We consider a quantity of interest $Q$ which is supposed to be the goal of the computation. Performing an optimal calibration process with respect to $Q$ means: (i) updating relevant parameters only, i.e. those that affect $Q$; (ii) setting up relevant measurements, i.e. some for which $Q$ is sensitive.
A first approach to select relevant parameters with respect to the quantity of interest $Q$ would consist in obtaining the sensitivity of $Q$ with respect to $\mathbf{p}$, i.e. the gradient $\nabla_{\mathbf{p}} Q$. The relation between $Q$ and $\mathbf{p}$ depends on the mathematical model only; the updating ) process is not involved here. The quantity of interest thus reads $Q(\mathbf{u}(\mathbf{p}))$, and optimal control tools can be used by defining the Lagrangian:

$\mathcal{L}(\mathbf{p}, \mathbf{u}, \boldsymbol{\lambda})=Q(\mathbf{u})-\mathcal{A}(\mathbf{p}, \mathbf{u}, \boldsymbol{\lambda})$

Finding the saddle-point leads to a system similar to (8) (replacing $\mathcal{F}$ by $\mathcal{Q}$ ) and we directly get:

$$
\mathrm{d}_{\mathbf{p}} Q(\mathbf{u}(\mathbf{p}) ; \delta \mathbf{p})=\mathcal{L}_{\mathbf{p}}^{\prime}(\mathbf{p}, \mathbf{u}, \boldsymbol{\lambda} ; \delta \mathbf{p})=-\mathcal{A}_{\mathbf{p}}^{\prime}(\mathbf{p}, \mathbf{u}, \boldsymbol{\lambda} ; \delta \mathbf{p})
$$

where $\mathbf{u}(\mathbf{p})$ verifies the state equation (4) and $\boldsymbol{\lambda}(\mathbf{p})$ verifies the following co-state (or adjoint) equation:

$Q_{\mathbf{u}}^{\prime}(\mathbf{u} ; \delta \mathbf{u})-a(\mathbf{p}, \delta \mathbf{u}, \boldsymbol{\lambda})=0 \quad \forall \delta \mathbf{u} \in \mathcal{U}_{0}$

Remark 5 If $Q$ is linear with respect to $\mathbf{u}$, the first term of the adjoint problem reads $Q(\delta \mathbf{u})$.

Remark 6 The adjoint solution (27), valid for any loading of the direct problem but for a given set of parameters $\mathbf{p}$, also enables to address goal-oriented discretization error estimation in the prediction problem. This gives information to define an optimal finite element mesh for the prediction of the quantity of interest.

Normalized terms $w_{Q, i}=p_{i} \frac{\partial Q}{\partial p_{i}}$ could be used as weighting factors in the localization step, in order to give weight to parameters with are influent for $Q$. However, this strategy is debatable as information coming from gradient values may not be optimal and reliable to choose parameters to update and to define a stopping criterion in the minimization process.

In the following, we rather choose to define an automatic process in which new cost functions related to the constitutive relation error and dedicated to the quantity of interest $Q$ are minimized.

4.1 Goal-oriented cost function when the quantity of interest is not measured

We assume here that the spatial region in which $Q$ is defined is not a measurement point. Keeping the philosophy and flexibility of the modified CRE, composed 
of modeling and measurements error terms, we introduce a new cost function, denoted $\mathcal{F}_{Q}(\mathbf{p})$, associated with the considered quantity of interest. It reads:

$\mathcal{F}_{Q}(\mathbf{p})=\min _{q \in \mathbb{R}}\left[\frac{1}{2}\left|q-Q_{\text {mod }}(\mathbf{p})\right|^{2}+\frac{1}{2} \frac{r}{1-r}\left|q-Q_{o b s}(\mathbf{p})\right|^{2}\right]$

The modeling error term $\frac{1}{2}\left|q-Q_{\bmod }(\mathbf{p})\right|^{2}$ involves a value $Q_{\text {mod }}$ of the quantity of interest defined from the model only, i.e. by means of state equation (4):

$Q_{\text {mod }}(\mathbf{p})=Q\left(\mathbf{u}_{1}(\mathbf{p})\right) \quad ; \quad \mathcal{A}\left(\mathbf{p}, \mathbf{u}_{1}, \mathbf{v}\right)=0 \quad \forall \mathbf{v} \in \mathcal{U}_{0}$

After discretization, $\mathbf{u}_{1}$ is thus obtained by finding $\mathbf{U}_{1} \in$ $\mathcal{U}^{h}$ solution of the system:

$\widetilde{\mathbb{K}}(\mathbf{p}) \mathbf{U}_{1}=\widetilde{F}$

The measurement error term $\frac{1}{2}\left|q-Q_{o b s}(\mathbf{p})\right|^{2}$ involves a value $Q_{o b s}$ of the quantity of interest defined from an interpolation of measurements $\mathbf{s}_{o b s}$, i.e.:

$Q_{\text {obs }}(\mathbf{p})=Q\left(\mathbf{u}_{2}(\mathbf{p})\right) \quad ; \quad\left(\mathbf{u}_{2}, \sigma_{2}\right)=\underset{(\mathbf{v}, \pi) \in \mathcal{U} \times \mathcal{S}}{\operatorname{argmin}} \mathcal{E}_{m}^{2}(\mathbf{p}, \mathbf{v}, \pi)$

The definition of $\mathbf{u}_{2}$ thus involves $\mathbf{s}_{o b s}$ and leads to a system $\overline{\mathbb{K}}(\mathbf{p}) \mathbf{U}_{2}=\overline{\mathbf{F}}$ after discretization (cf. (22)).

Remark 7 If several experiments are performed, with several loadings for instance, the cost function may be defined from a sum of individual components, each involving modeling and measurement terms and associated with a given experiment.

Remark 8 In cases where measurements of $Q$ are available, these should be directly used as $Q_{o b s}$ in (28) so that the regularization process brought by $\mathcal{E}_{m}$ is no more used in $\mathcal{F}_{Q}$. This specific case will be studied in the following section.

The computation of $\mathcal{F}_{Q}(\mathbf{p})$ (or its discretized version $\left.\mathcal{F}_{Q}^{h}(\mathbf{p})\right)$ is trivial and we obtain:

$$
\begin{aligned}
q & =(1-r) Q\left(\mathbf{U}_{1}\right)+r Q\left(\mathbf{U}_{2}\right) \\
\mathcal{F}_{Q}^{h}(\mathbf{p}) & =\frac{1}{2} r\left[Q\left(\mathbf{U}_{1}\right)-Q\left(\mathbf{U}_{2}\right)\right]^{2}
\end{aligned}
$$

with $\mathbf{U}_{1}$ (resp. $\mathbf{U}_{2}$ ) verifying (30) (resp. (22)). The gradient of $\mathcal{F}_{Q}^{h}(\mathbf{p})$ is computed using the adjoint state method; we introduce for that the discrete Lagrangian:

$$
\begin{aligned}
\mathcal{L}_{Q}\left(\mathbf{p}, \mathbf{U}_{1}, \mathbf{U}_{2}, \boldsymbol{\Lambda}_{1}, \boldsymbol{\Lambda}_{2}\right) & =\frac{1}{2} r\left[Q\left(\mathbf{U}_{1}\right)-Q\left(\mathbf{U}_{2}\right)\right]^{2} \\
& -\boldsymbol{\Lambda}_{1}^{T}\left(\widetilde{\mathbb{K}}(\mathbf{p}) \mathbf{U}_{1}-\widetilde{\mathbf{F}}\right) \\
& -\boldsymbol{\Lambda}_{2}^{T}\left(\overline{\mathbb{K}}(\mathbf{p}) \mathbf{U}_{2}-\overline{\mathbf{F}}\right)
\end{aligned}
$$

Finding the saddle-point of $\mathcal{L}_{Q}$ leads to verify, in addition to conditions from (29) (resp. (31)) for $\mathbf{U}_{1}$ (resp. $\left.\mathbf{U}_{2}\right)$, and for all $\left(\delta \mathbf{U}_{1}, \delta \mathbf{U}_{2}\right) \in \mathcal{U}_{0}^{h} \times \mathcal{U}_{0}^{h}$ :

$\boldsymbol{\Lambda}_{1}^{T} \widetilde{\mathbb{K}}(\mathbf{p}) \delta \mathbf{U}_{1}=\beta Q^{\prime}\left(\mathbf{U}_{1} ; \delta \mathbf{U}_{1}\right) \quad \forall \delta \mathbf{U}_{1} \in \mathcal{U}_{0}^{h}$

$\boldsymbol{\Lambda}_{2}^{T} \overline{\mathbb{K}}(\mathbf{p}) \delta \mathbf{U}_{2}=-\beta Q^{\prime}\left(\mathbf{U}_{2} ; \delta \mathbf{U}_{2}\right) \quad \forall \delta \mathbf{U}_{2} \in \mathcal{U}_{0}^{h}$

with $\beta=r\left[Q\left(\mathbf{U}_{1}\right)-Q\left(\mathbf{U}_{2}\right)\right]$.

The gradient of $\mathcal{F}_{Q}^{h}(\mathbf{p})$ can then be computed using the adjoint solutions $\boldsymbol{\Lambda}_{1}$ and $\boldsymbol{\Lambda}_{2}$ :

$$
\begin{aligned}
\mathrm{d}_{\mathbf{p}} \mathcal{F}_{Q}^{h}(\mathbf{p} ; \delta \mathbf{p}) & =\mathcal{L}_{Q, \mathbf{p}}^{\prime}\left(\mathbf{p}, \mathbf{U}_{1}, \mathbf{U}_{2}, \boldsymbol{\Lambda}_{1}, \boldsymbol{\Lambda}_{2} ; \delta \mathbf{p}\right) \\
& =-\boldsymbol{\Lambda}_{1}^{T} \mathrm{~d}_{\mathbf{p}} \widetilde{\mathbb{K}}\left(\mathbf{p} ; \delta_{\mathbf{p}}\right) \mathbf{U}_{1}-\boldsymbol{\Lambda}_{2}^{T} \mathrm{~d}_{\mathbf{p}} \overline{\mathbb{K}}\left(\mathbf{p} ; \delta_{\mathbf{p}}\right) \mathbf{U}_{2}
\end{aligned}
$$

Elements of $\mathbf{p}$ associated with high components for the gradient of $\mathcal{F}_{Q}^{h}(\mathbf{p})$ are then selected to drive the nonlinear hierarchical minimization, and iterations are stopped when the value of $\mathcal{F}_{Q}^{h}$ reaches a given tolerance value. Consequently, this method enables to drive the updating process in an optimal and natural manner with respect to the quantity of interest.

4.2 Goal-oriented cost function when the quantity of interest is measured

In this specific case, there is no interpolation of the data and therefore the regularization term involved in the previous cost function is missing. We propose a new cost function based on a local version of the modified CRE, i.e. a measure at point (or in the area) where the quantity of interest is defined. We denote by $Q^{*}$ the dual quantity of $Q$ (in the energy sense), and we write the local constitutive relation between $Q^{*}$ and $Q$ under the form $Q^{*}=k(\mathbf{p}) Q$. The new cost function thus reads:

$\mathcal{F}_{Q}(\mathbf{p})=\min _{(\mathbf{v}, \pi) \in \mathcal{U} \times \mathcal{S}} \mathcal{E}_{m, l o c}^{2}(\mathbf{p}, \mathbf{v}, \pi)$

with

$$
\begin{aligned}
\mathcal{E}_{m, l o c}^{2}(\mathbf{p}, \mathbf{v}, \pi) & =\frac{1}{2}\left|Q^{*}(\pi)-k(\mathbf{p}) Q(\mathbf{v})\right|^{2} \\
& +\frac{1}{2} \frac{r}{1-r}\left|Q(\mathbf{v})-Q_{o b s}\right|^{2}
\end{aligned}
$$


The two terms in $\mathcal{E}_{m, l o c}^{2}$ correspond to local modeling and measurement errors, respectively. Following a procedure similar to the one detailed in Section 3.2, we write the discrete version of (37):

$$
\begin{aligned}
\mathcal{E}_{m, l o c}^{h 2}(\mathbf{p}, \mathbf{U}, \mathbf{V}) & =\frac{1}{2}\left|Q^{*}(\mathbf{V})-k(\mathbf{p}) Q(\mathbf{U})\right|^{2} \\
& +\frac{1}{2} \frac{r}{1-r}\left|Q(\mathbf{U})-Q_{o b s}\right|^{2}
\end{aligned}
$$

with $\mathbb{K}(\mathbf{p}) \mathbf{V}=\mathbf{F}$ (equilibrium), and the computation of $\mathcal{F}_{Q}^{h}(\mathbf{p})$ is performed by searching the saddle-point of the following lagrangian:

$\mathcal{L}_{Q}(\mathbf{p}, \mathbf{U}, \mathbf{V}, \boldsymbol{\Lambda})=\mathcal{E}_{m, l o c}^{h 2}(\mathbf{p}, \mathbf{U}, \mathbf{V})-\boldsymbol{\Lambda}^{T}(\mathbb{K}(\mathbf{p}) \mathbf{V}-\mathbf{F})$

This leads to the system, for all $\delta \mathbf{V} \in \mathcal{U}_{\mathcal{S}}^{h}$ :

$$
\begin{array}{r}
-k(\mathbf{p})\left(Q^{*}(\mathbf{V})-k(\mathbf{p}) Q(\mathbf{U})\right)+\frac{r}{1-r}\left(Q(\mathbf{U})-Q_{o b s}\right)=0 \\
Q_{\mathbf{u}}^{*^{\prime}}(\mathbf{V} ; \delta \mathbf{V})\left(Q^{*}(\mathbf{V})-k(\mathbf{p}) Q(\mathbf{U})\right)-\Lambda^{T} \mathbb{K}(\mathbf{p}) \delta \mathbf{V}=0 \\
\mathbb{K}(\mathbf{p}) \mathbf{V}-\mathbf{F}=\mathbf{0}
\end{array}
$$

The second equation (40) is the discretized version of an adjoint problem; combining the other two provides for the system that $\mathbf{U}$ should verify.

The evaluation of the gradient of $\mathcal{F}_{Q}^{h}(\mathbf{p})$ is then direct with the adjoint state method:

$\mathrm{d}_{\mathbf{p}} \mathcal{F}^{h}(\mathbf{p} ; \delta \mathbf{p})=\mathcal{L}_{\mathbf{p}}^{\prime}(\mathbf{p}, \mathbf{U}, \mathbf{V}, \boldsymbol{\Lambda} ; \delta \mathbf{p})$

with $(\mathbf{U}, \mathbf{V}, \boldsymbol{\Lambda})$ solution of (40). Here again, elements of $\mathbf{p}$ associated with high components of the gradient are selected in order to minimize $\mathcal{F}_{Q}^{h}(\mathbf{p})$ with respect to these parameters only. Iterations of the updating process are continued until a tolerance value is reached.

\subsection{Remarks on the regularization process}

In the goal-oriented updating method involving cost functions $\mathcal{F}_{Q}$ introduced in Sections 4.1 or 4.2 , it is important to notice that an iterative two-step (localization, correction) strategy is conserved. However, the localization step is no more based on a splitting of the modeling error term and comparison between parameter contributions $\left(\mathcal{F}_{Q}\right.$ is local in space) as used in Section 3.2 , but on the selection of parameters that bring highest components to the gradient of $\mathcal{F}_{Q}(\mathbf{p})$. In practice, at each iteration of the method, the parameter (or the set of parameters) that brings the highest gradient component is corrected first. If this correction is not associated with a significant decrease of the cost function value, the parameter (or the set of parameters) that brings the second highest gradient component is corrected, and the process is stopped when a significant decrease of $\mathcal{F}_{Q}(\mathbf{p})$ is observed. This hierarchical strategy naturally regularizes the goal-oriented inverse formulation.

\section{Sensitivity with respect to measurements}

We now wish to obtain the sensitivity of $Q$ with respect to measurements, i.e. the relation between a change in observed values $\mathbf{s}_{\text {obs }}$ and the output quantity $Q$, in order to get useful information for setting up optimal measurements. The updating process is involved here, as a change in $\mathbf{s}_{\text {obs }}$ affects the value of $\mathbf{p}$. The calibration process (17) using the modified CRE can be seen as a constrained minimization problem (with fixed measurements $\mathbf{s}_{o b s}$ ), its solution being the saddle-point of the following Lagrangian:

$$
\begin{aligned}
& \mathcal{L}(\mathbf{q}, \mathbf{v}, \pi, \boldsymbol{\mu})=\mathcal{E}_{m}^{2}(\mathbf{q}, \mathbf{v}, \pi) \\
& -\left[\int_{\Omega} \pi: \notin(\boldsymbol{\mu}) \mathrm{d} \Omega-\int_{\Omega} \mathbf{f}_{d} \cdot \boldsymbol{\mu} \mathrm{d} \Omega-\int_{\partial_{2} \Omega} \mathbf{F}_{d} \cdot \boldsymbol{\mu} \mathrm{d} S\right]
\end{aligned}
$$

$\mathcal{L}$ explicitly depends on measurements $\mathbf{s}_{\text {obs }}$ which are involved in the definition of $\mathcal{E}_{m}$, so that we can also write $\mathcal{L}\left(\mathbf{q}, \mathbf{v}, \pi, \boldsymbol{\mu}, \mathbf{s}_{\text {obs }}\right)$.

As regards the quantity of interest, it reads $Q\left(\mathbf{z}\left(\mathbf{s}_{\text {obs }}\right)\right)$ with $\mathbf{z}=(\mathbf{p}, \mathbf{u}, \sigma, \boldsymbol{\lambda})$ solution of the calibration problem, i.e. realizing the saddle-point of $\mathcal{L}$ :

$\mathcal{L}_{\mathbf{z}}^{\prime}\left(\mathbf{z}, \mathbf{s}_{\text {obs }} ; \delta \mathbf{z}\right)=0 \quad \forall \delta \mathbf{z}$

In order to compute the gradient of $Q$ with respect to $\mathbf{s}_{\text {obs }}$, we use again optimal control tools and introduce the Lagrangian $\mathcal{H}$ defined as:

$\mathcal{H}\left(\mathbf{z}, \mathbf{s}_{o b s}, \mathbf{y}\right)=Q(\mathbf{z})-\mathcal{L}_{\mathbf{z}}^{\prime}\left(\mathbf{z}, \mathbf{s}_{o b s} ; \mathbf{y}\right)$

Finding the solution $\mathbf{y}$ of the adjoint problem:

$\mathcal{L}_{\mathbf{z z}}^{\prime \prime}\left(\mathbf{z}, \mathbf{s}_{o b s} ; \mathbf{y}, \delta \mathbf{z}\right)=Q_{\mathbf{z}}^{\prime}(\mathbf{z} ; \delta \mathbf{z}) \quad \forall \delta \mathbf{z}$

enables to obtain the following sensitivity result:

$$
\begin{aligned}
\mathrm{d}_{\mathbf{s}_{o b s}} Q\left(\mathbf{z}\left(\mathbf{s}_{o b s}\right) ; \delta \mathbf{s}_{o b s}\right) & =\mathcal{H}_{\mathbf{s}_{o b s}}^{\prime}\left(\mathbf{z}, \mathbf{s}_{o b s}, \mathbf{y} ; \delta \mathbf{s}_{o b s}\right) \\
& =-\mathcal{L}_{\mathbf{z} \mathbf{s}_{o b s}}^{\prime \prime}\left(\mathbf{z}, \mathbf{s}_{o b s} ; \mathbf{y}, \delta \mathbf{s}_{o b s}\right)
\end{aligned}
$$

with $\mathbf{z}$ verifying (43). 
Remark 9 This same adjoint solution $\mathbf{y}$, with fixed measurements $\mathbf{s}_{\text {obs }}$, also enables to address sensitivity with respect to the finite element mesh used for calibration that may have influence on the solution of the optimization problem and identified parameters $\mathbf{p}$ (see [6]). Denoting by $\mathbf{z}_{h}$ the approximate solution of $\mathbf{z}$ obtained with the finite element method, the discretization error on an output of interest reads $Q(\mathbf{z})-Q\left(\mathbf{z}_{h}\right)=\mathcal{R}\left(\mathbf{z}_{h} ; \mathbf{y}\right)$, with $\mathcal{R}$ the residual functional, and an error estimate can be computed with a higher order approximation of the adjoint solution $\mathbf{y}$.

Remark 10 Choosing $Q=p_{i}$ in the sensitivity analysis enables to measure the effect of uncertainty on observed values on calibrated parameter values.

Considering a discrete version of the Lagrangian $\mathcal{L}$ shows that the computational effort to obtain $d_{\mathbf{s}_{o b s}} Q$ is reasonable. The discretized version of (42) is similar to (62), and searching its saddle-point leads to:

$$
\begin{aligned}
B\left(\mathbf{p}, \mathbf{U}_{o b s} ; \delta \mathbf{p}\right) & \equiv \frac{1}{2}(\mathbf{U}-\mathbf{V})^{T} \mathrm{~d}_{\mathbf{p}} \mathbb{K}(\mathbf{p} ; \delta \mathbf{p})(\mathbf{U}+\mathbf{V}) \\
& =0 \quad \forall \delta \mathbf{p} \in \mathcal{P}_{0}
\end{aligned}
$$

with $\mathbf{U}$ and $\mathbf{V}$ given by (21) and depending on $\mathbf{p}$. After introducing the discrete Lagrangian:

$\mathcal{H}\left(\mathbf{p}, \mathbf{U}_{o b s}, \mathbf{q}\right)=Q(\mathbf{U}(\mathbf{p}))-B\left(\mathbf{p}, \mathbf{U}_{o b s} ; \mathbf{q}\right)$

and solving the associated adjoint problem:

$B_{\mathbf{p}}^{\prime}\left(\mathbf{p}, \mathbf{U}_{o b s} ; \mathbf{q}, \delta \mathbf{p}\right)=Q_{\mathbf{p}}^{\prime}(\mathbf{U}(\mathbf{p}) ; \delta \mathbf{p}) \quad \forall \delta \mathbf{p} \in \mathcal{P}_{0}$

we get:

$$
\begin{aligned}
\mathrm{d}_{\mathbf{U}_{o b s} Q\left(\mathbf{U}\left(\mathbf{p}, \mathbf{U}_{o b s}\right) ; \delta \mathbf{U}_{o b s}\right)}=\mathcal{H}_{\mathbf{U}_{o b s}}^{\prime}\left(\mathbf{p}, \mathbf{U}_{o b s}, \mathbf{q}, \delta \mathbf{U}_{o b s}\right) \\
=-B_{\mathbf{U}_{o b s}}^{\prime}\left(\mathbf{p}, \mathbf{U}_{o b s} ; \mathbf{q}, \delta \mathbf{U}_{o b s}\right)
\end{aligned}
$$

with U (resp. q) verifying (22) (resp. (49)).

The gradient $\mathrm{d}_{\mathbf{U}_{o b s}} Q$ can be computed at each iteration of the updating process from available information and after solving (49). The optimization of the experimental protocol thus consists in finding sensor positions and measurements that provide for maximal values of $\left|s_{o b s}^{(i)} \mathrm{d}_{s_{o b s}^{(i)}} Q\right|$ or $\left|U_{o b s}^{(i)} \mathrm{d}_{U_{o b s}^{(i)}} Q\right|\left(i=1, \ldots, n_{o b s}\right)$.

\section{Numerical results}

\subsection{Trussed structure}

We first illustrate the method on a simple trussed structure with lattice beams represented in Figure 2. It is composed of 10 beams for which respective Young's moduli $E_{i}$ are parameters that need to be updated. The loading, perfectly known, consists of a pointwise vertical force applied at point $P$. We assume that the quantity of interest in the study of this structure is the vertical displacement of point $P\left(Q(\mathbf{u})=u_{y}(P)\right)$.

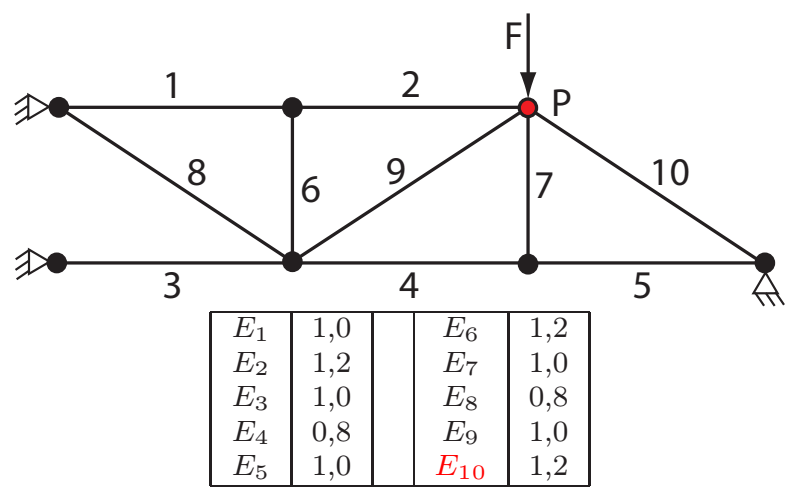

Fig. 2 Considered trussed structure (left) and reference values for the material parameters (right).

Measurements consist of nodal displacements (eight scalar data) over the whole structure; their value is simulated considering a reference model in which stiffness parameters $E_{i}$ are set to the value indicated in Figure 2 (no noise). The initial model to be updated is defined by a $50 \%$ perturbation of parameters, i.e. $E_{i}^{*}=1,5 \times E_{i}$ $(i=1, \ldots, 10)$. The updating process is conducted using a gradient method, with constant path, on a functional $\mathcal{F}_{Q}$ which is a combination of the one proposed in Section 4.2 (related to measurement at node $P$ ) and the one proposed in Section 4.1 (related to other measurements). The minimization is performed on one parameter only at each iteration.

Figure 3 shows values of $Q$ which are predicted by the model after each updating iteration, with both classical updating (global constitutive relation error) and updating dedicated to $Q$ and with the same experimental data. We observe that the new updating strategy provides, after a few iterations only, for a good quality model as regards the prediction of $Q$.

We show on Figure 4 the ratio $E_{i}^{*} / E_{i}$ (updated parameter over reference parameter) after 10 updating iterations, both for classical updating and updating dedicated to $Q$. We clearly observe that in the second case, 


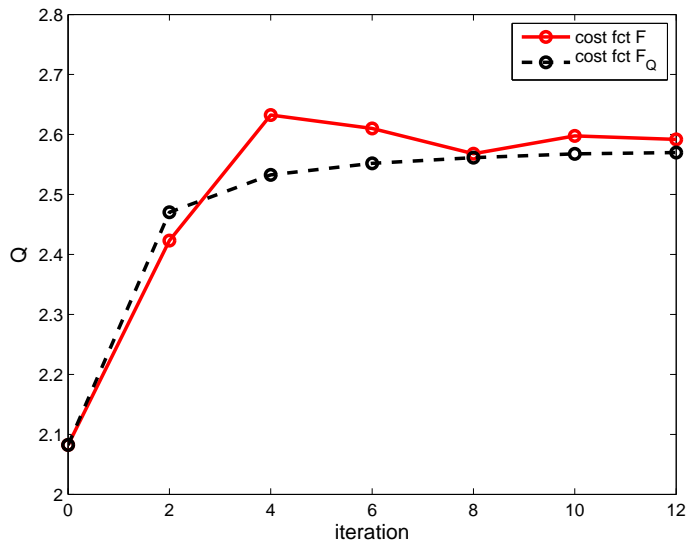

Fig. 3 Comparison of values of $Q$ predicted by the model after each updating iteration.

the updating strategy favors a subset of parameters which have a large influence on the predicted value of $Q$. This illustrates the fact that, compared to the classical one, the goal-oriented updating method analyzes in a different manner the amount of available experimental information.
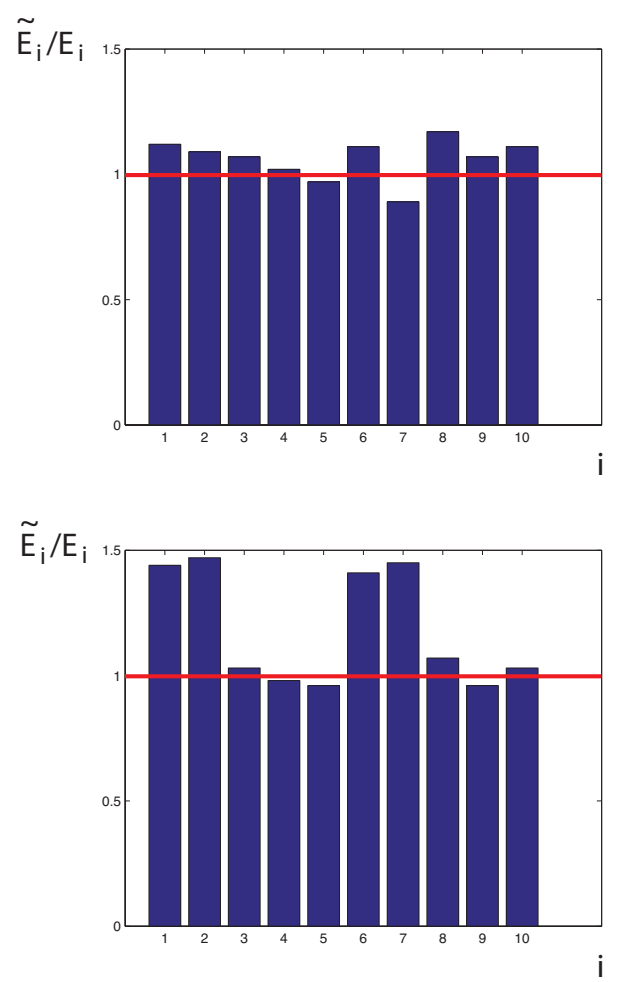

Fig. 4 Ratio $E_{i}^{*} / E_{i}$ after iteration 10 in the updating process, for classical updating (left) and updating dedicated to $Q$ (right).

\subsection{Two-dimensional beam structure}

We now consider a $2 \mathrm{D}$ concrete beam with a steel bar $\Gamma$ (see Figure 5). In civil engineering applications, concrete Young modulus $E_{c}$ may decrease due to damage, the steel bar cross-section $S_{b}$ may be reduced due to corrosion effects, and the rigidity $k$ of the supports may not be well-known. We divide the steel bar $\Gamma$ (resp. the lower concrete part $\Omega_{c}$ ) into five subdomains $\Gamma_{j}$ (resp. $\Omega_{c_{j}}$ ), $j \in\{1, . ., 5\}$ (see Figure 5) and consider piecewise constant steel bar cross-section and concrete Young's modulus. We denote by $S_{b_{j}}$ the steel bar crosssection in $\Gamma_{j}, E_{c_{j}}$ the concrete Young modulus in $\Omega_{c_{j}}$, and $k_{1}$ (resp. $k_{2}$ ) the rigidity of support 1 (resp. support 2). We take $L=30 \mathrm{~m}$ and $H=1 \mathrm{~m}$.

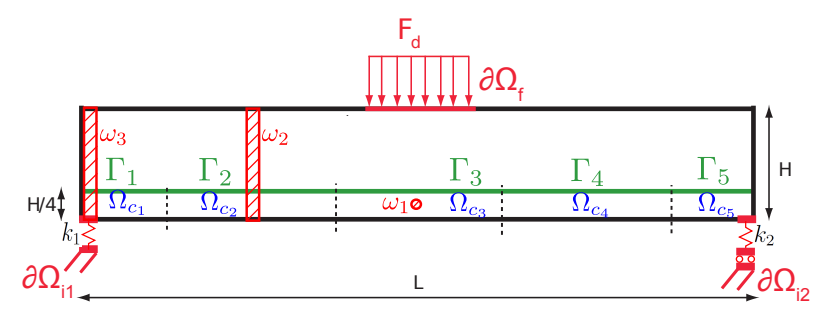

Fig. 5 2D concrete beam with a horizontal steel bar, with domains of interest $\omega_{1}, \omega_{2}$ and $\omega_{3}$.

To update beam parameters $\left(\left\{S_{b_{j}}\right\},\left\{E_{c_{j}}\right\}, k_{1}, k_{2}\right)$, a static loading $\mathbf{F}_{d}$ is applied and the longitudinal strain component $\epsilon_{x x}$ is measured at 14 points (see Figure 6). The displacement field $\mathbf{u} \in \mathcal{U}_{0}=\left\{\mathbf{u}^{*} \in H_{1}(\Omega) \backslash \mathbf{u}^{*} \cdot \mathbf{x}=\right.$ 0 on $\left.\partial \Omega_{i 1}\right\}$ satisfies the following direct problem:

$$
\begin{aligned}
& \int_{\Omega} \mathbb{E}(\mathbf{u}): \boldsymbol{K}_{c} \mathbb{E}\left(\mathbf{u}^{*}\right) d \Omega+\int_{\Gamma} E_{b} S_{b} \frac{\partial u_{x}}{\partial x} \frac{\partial u_{x}^{*}}{\partial x} d \Gamma \\
& +\int_{\Omega_{i 1}} k_{1} u_{y} u_{y}^{*} d \Gamma+\int_{\Omega_{i 2}} k_{2} u_{y} u_{y}^{*} d \Gamma-\int_{\partial \Omega_{f}} \mathbf{F}_{d} \cdot \mathbf{u}^{*} \partial \Omega \\
& =0, \forall \mathbf{u}^{*} \in \mathcal{U}_{0}
\end{aligned}
$$

where $\boldsymbol{K}_{c}$ represents Hooke tensor depending on concrete Young's moduli $E_{c_{j}}(j \in\{1, . ., 5\})$.

The sensor outputs are simulated numerically considering the reference beam parameters defined below and direct model (51). In the reference model, let us notice that a loss of stiffness is considered in subdomain $\Omega_{c_{3}}$.

$$
\left\{\begin{array}{l}
E_{c_{1}}^{r e f}=E_{c_{2}}^{r e f}=E_{c_{4}}^{r e f}=E_{c_{5}}^{r e f}=40.10^{9} \mathrm{~Pa} \\
E_{c_{3}}^{r e f}=30.10^{9} \mathrm{~Pa} \\
S_{b_{1}}^{r e f}=S_{b_{2}}^{r e f}=S_{b_{3}}^{r e f}=S_{b_{4}}^{r e f}=S_{b_{5}}^{r e f}=0.04 \mathrm{~m} \\
k_{1}^{r e f}=k_{2}^{r e f}=5.10^{7} \mathrm{~N} / \mathrm{m}^{3}
\end{array}\right.
$$


In the initial model to be updated, we take the undamaged standard value of the concrete Young moduli $\left(E_{c_{j}}=40.10^{9} P a, j \in\{1, . ., 5\}\right)$, the value of the uncorroded steel bar cross-section $\left(S_{b_{j}}=0.04 \mathrm{~m}, j \in\right.$ $\{1, . ., 5\})$ and the standard value of the rigidity $\left(k_{1}=\right.$ $k_{2}=5.10^{7} \mathrm{~N} / \mathrm{m}^{3}$ ). The only difference between the reference and the initial models is the value of the concrete Young modulus in subdomain $\Omega_{c_{3}}$.

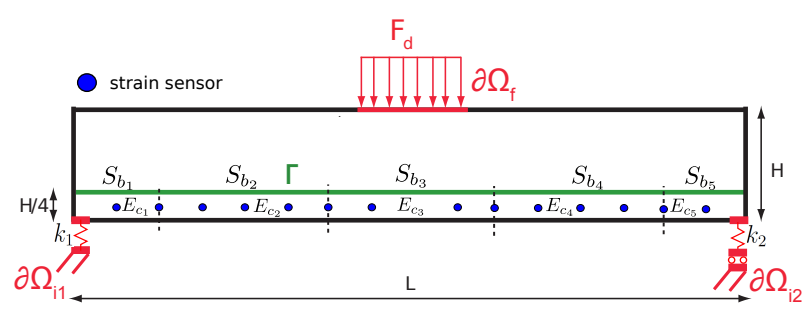

Fig. 6 Instrumentation of the beam with 14 strain sensors.

The goal-oriented updating procedure is applied to three quantities of interest:

$Q_{1}=\int_{\omega_{1}} \epsilon_{x x} d \Omega ; \quad Q_{2}=\int_{\omega_{2}} u_{y} d \Omega ; \quad Q_{3}=\int_{\omega_{3}} u_{y} d \Omega$

The associated zones of interest $\omega_{1}, \omega_{2}$ and $\omega_{3}$ are represented in Figure 5.

Regarding the updating strategy, the highest component of the functional gradient is searched at each iteration and the associated parameter is updated. When the value of the functional does not decrease after updating the parameter, the initial parameter value is kept and the parameter having the second highest functional gradient is updated, and so on ... When the value of the quantity of interest remains unchanged (variation less than $0.1 \%$ ) between two successive iterations, the updating algorithm is stopped.

For each iteration of the goal-oriented technique, the updated parameter values and associated predicted values of the quantity of interest are represented in Figures 7 and 8 . Several observations can be made:

- initially, we observe that the error between the quantity of interest and the reference quantity of interest is the highest for $Q_{1}$; it is about $15 \%$;

- as regards quantities of interest $Q_{1}$ and $Q_{2}$, two iterations are sufficient to get an error less than $1 \%$ on the determination of the concrete Young modulus $E_{c_{3}}$. We can remark that only parameter $E_{c_{3}}$ has been updated using the goal-oriented approach.;
- as regards quantity $Q_{3}$, the updating procedure suggests a 20\% reduction of the concrete Young modulus $E_{c_{2}}$ at iteration 1 . The modification of this parameter leading to a variation of the quantity of interest less than $0.1 \%$, we do not take into account the modification of $E_{c_{2}}$ and the updating algorithm is stopped;

- quantity $Q_{3}$ being not sensitive to $E_{c_{3}}$, this parameter is not updated using the goal-oriented approach. Indeed, we see in Figure 8 that the ratio of the non-updated and the reference value of quantity $Q_{3}$ is almost 1 . Parameter updating is thus useless regarding quantity of interest $Q_{3}$.

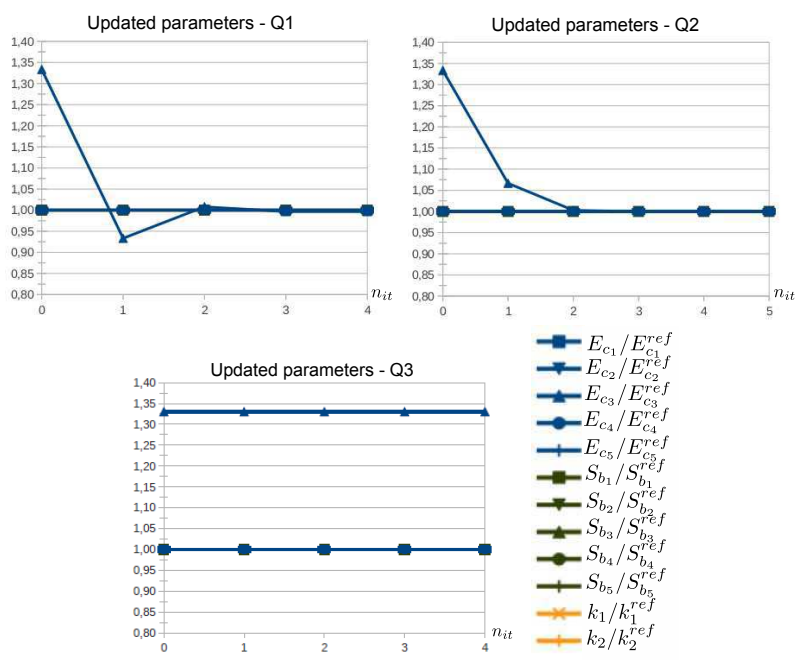

Fig. 7 Updated beam parameters at each iteration of the updating goal-oriented approach for quantities of interest $Q_{1}$, $Q_{2}$, and $Q_{3}$.

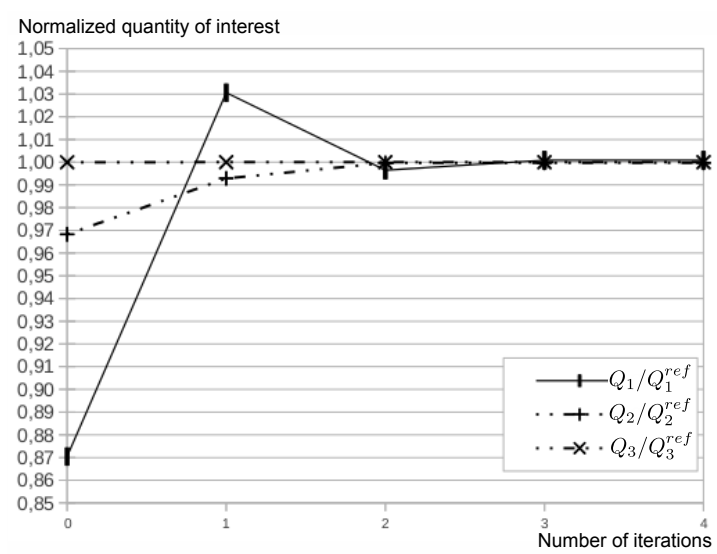

Fig. 8 Ratio between the updated and the reference values of the quantity of interest at each iteration of the updating goal-oriented approach for $Q_{1}, Q_{2}$ and $Q_{3}$. 


\subsection{D thermal problem in a building}

In thermal building applications, multizone models are commonly used [23]. In such models, a building is decomposed into zones and envelopes. The temperature in each zone is supposed homogeneous and verifies an ODE in time; the temperature in each envelope satisfies a one-dimensional PDE. An inverse modeling technique has been proposed in [30] to identify envelope parameters and the thermal loading. Herein, we deal with another kind of inverse modeling technique based on the constitutive relation error framework.

We consider a thermal building problem, represented in Figure 9, with two zones $\mathrm{A}$ and $\mathrm{B}$ separated by a wall $\mathrm{W}$ (envelope). The temperature in zones A and B (resp. in the wall W) are denoted $T_{A}(t)$ and $T_{B}(t)$ (resp. $\left.T_{W}(x, t)\right)$. As for the mechanical problem presented in Section 2.1, the unsteady thermal problem can be written using the constitutive relation error formalism:

- initial conditions

$$
\begin{aligned}
T_{A}(t=0) & =T_{A}^{0} \\
T_{W}(x, t=0) & =T_{W}^{0}(x), x \in[0, L] \\
T_{B}(t=0) & =T_{B}^{0}
\end{aligned}
$$

- balance equations

$$
\begin{aligned}
c_{A} \frac{d T_{A}}{d t}-q_{A} & =w_{A}, t \in[0, T] \\
c_{W} \frac{\partial T_{W}}{\partial t}+\frac{\partial q}{\partial x} & =0, x \in[0, L], t \in[0, T] \\
c_{B} \frac{d T_{B}}{d t}-q_{B} & =w_{B}, t \in[0, T] \\
q+q_{A} & =0, x=0, t \in[0, T] \\
-q+q_{B} & =0, x=L, t \in[0, T]
\end{aligned}
$$

- constitutive equations

$$
\begin{aligned}
q & =-d_{W} \frac{\partial T_{W}}{\partial x}, x \in[0, L], t \in[0, T] \\
q_{A} & =\alpha_{A}\left(T_{W}(x=0)-T_{A}\right), x=0, t \in[0, T] \\
q_{B} & =\alpha_{B}\left(T_{W}(x=L)-T_{B}\right), x=L, t \in[0, T]
\end{aligned}
$$

where $c_{A}$ (resp. $\left.c_{B}\right)\left(J . K^{-1}\right)$ represents the overall heat capacity of zone A (resp. zone B), $c_{W}\left(J . K^{-1} . m^{-1}\right)$ represents the overall heat capacity per meter of the wall, $\alpha_{A}$ (resp. $\left.\alpha_{B}\right)\left(W . K^{-1}\right)$ is the product of the surface envelope and the convective exchange coefficient between the wall and zone A (resp. zone B), $w_{A}$ (resp. $\left.w_{B}\right)(W)$ is the heating in zone $\mathrm{A}$ (resp. zone $\mathrm{B}$ ), and $d_{W}\left(W . m . K^{-1}\right)$ represents the overall conductivity per meter of the wall. We take $L=0.2 \mathrm{~m}$ and $T=43200 \mathrm{~s}$ (half a day) in the following.

We are interested in identifying the thermal model parameters $\mathbf{p}=\left\{c_{A}, \alpha_{A}, c_{W}, d_{W}, \alpha_{B}, c_{B}\right\}$. To achieve this purpose, two strategies are employed and compared: the first one is the classical calibration method based on the constitutive relation error; the second one is the goal-oriented updating introduced in Section 4. A detailed review of the extension of both strategies to the unsteady case is given in Appendix A.

In the goal-oriented approach, we consider the quantity of interest defined by:

$Q=\left\langle T_{W}(x=0, t)\right\rangle_{\Delta T} \quad ; \quad \Delta T=\left[\frac{11}{12} T, T\right]$

where $\langle\bullet\rangle_{\Delta T}$ denotes the time average over $\Delta T$. Let us note that $Q$ is a non-measured quantity of interest.

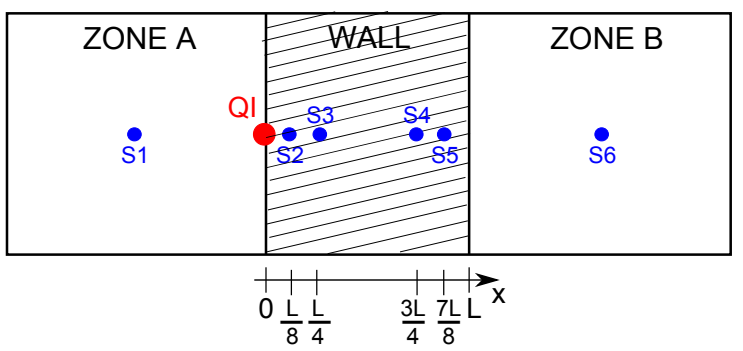

Fig. 9 Geometry of the unsteady thermal problem with two zones and one wall. $S_{i}$ denotes the $i^{\text {th }}$ temperature sensor and $Q I$ the quantity of interest.

Data consist in temperature values measured at 6 points spread along the longitudinal axis of the structure (Figure 9). To simulate the sensor outputs numerically, we solve equations (54-56) (using a classical finite element code) with reference parameters $c_{A}^{r e f}=$ $c_{B}^{r e f}=62500 J \cdot K^{-1}, c_{W}^{r e f}=2.10^{7} J \cdot K^{-1} \cdot m^{-1}, d_{W}^{r e f}=$ $20 W \cdot m \cdot K^{-1} \alpha_{A}^{r e f}=\alpha_{B}^{r e f}=83 W \cdot K^{-1}$ and we extract the solution at the sensor nodes. For the heating in zones $\mathrm{A}$ and $\mathrm{B}$, we consider $w_{A}=500 \mathrm{~W}$ and $w_{B}=0 \mathrm{~W}$ on the time interval $[0, T]$. As regards the initial conditions, we take $T_{A}^{0}=T_{B}^{0}=T_{W}^{0}(x)=10^{\circ} \mathrm{C}$. The reference solution is noted $T^{\text {ref }}$ and the reference value of the quantity of interest is $Q^{r e f}=16.50$. In the following updating studies, we consider a $50 \%$ initial relative error on all parameters. 
6.3.1 Calibration method based on the constitutive relation error

The modified constitutive relation error associated to the unsteady thermal building model is given by

$$
\begin{aligned}
\mathcal{E}_{m}^{2}= & \frac{1}{2} \int_{0}^{T} \int_{0}^{L} d_{W}\left(\frac{\partial u_{W}}{\partial x}-\frac{\partial v_{W}}{\partial x}\right)^{2} d x d t \\
& +\frac{1}{2} \int_{0}^{T} \alpha_{A}\left(u_{W}(0)-v_{W}(0)-u_{A}+v_{A}\right)^{2} d t \\
& +\frac{1}{2} \int_{0}^{T} \alpha_{B}\left(u_{W}(L)-v_{W}(L)-u_{B}+v_{B}\right)^{2} d t \\
& +\frac{1}{2} \frac{r}{1-r} \sum_{i=1}^{n_{s}} \int_{0}^{T} g_{i}\left(T\left(x_{i}\right)-T_{o b s}\right)^{2} d t
\end{aligned}
$$

where $u$ (resp. $v$ ) denotes the kinematically admissible temperature (resp. the statically admissible temperature) and $n_{s}$ the number of sensors. In practice, we take $r=0.5$ and $g_{i}=1 W . K^{-1}$. The measure $\mathcal{E}_{m}^{2}$ given in (58) is made of a data misfit term and three errors in the constitutive law associated to the wall, zone $\mathrm{A}$ and zone B.

The classical calibration method based on the constitutive relation error is applied to the thermal building problem; technical details on its specificities for timedependent problems are given in Appendix A. Numerical results are reported in Figure 10. We notice that the modified constitutive relation error reaches a plateau after about 200 iterations. As a consequence, the calibration should be stopped. At iteration 200, one has $\mathcal{E}_{m}^{2} \approx 1 W . K . s$ which corresponds to a data misfit less than $0.1 \%$ and all parameters (except zone B parameters) are exactly determined. Most of the parameters being well-determined, it is not surprising to find that the updated quantity of interest is almost equal to the reference quantity of interest. Nevertheless, the main drawback of the classical calibration method is the computational cost. Indeed, this method updates most of the model parameters, that may lead to a large number of iterations.

\subsubsection{Goal-oriented updating with the constitutive relation error}

Considering the quantity of interest defined in (57), we now apply goal-oriented approach for model updating presented in Appendix A. We first study the case of non-corrupted sensor outputs. In Figure 12, we observe that the wall parameters $c_{W}$ and $d_{W}$ are correctly determined after 3 iterations only. The value of the goaloriented functional, given in Figure 11, is divided by 20
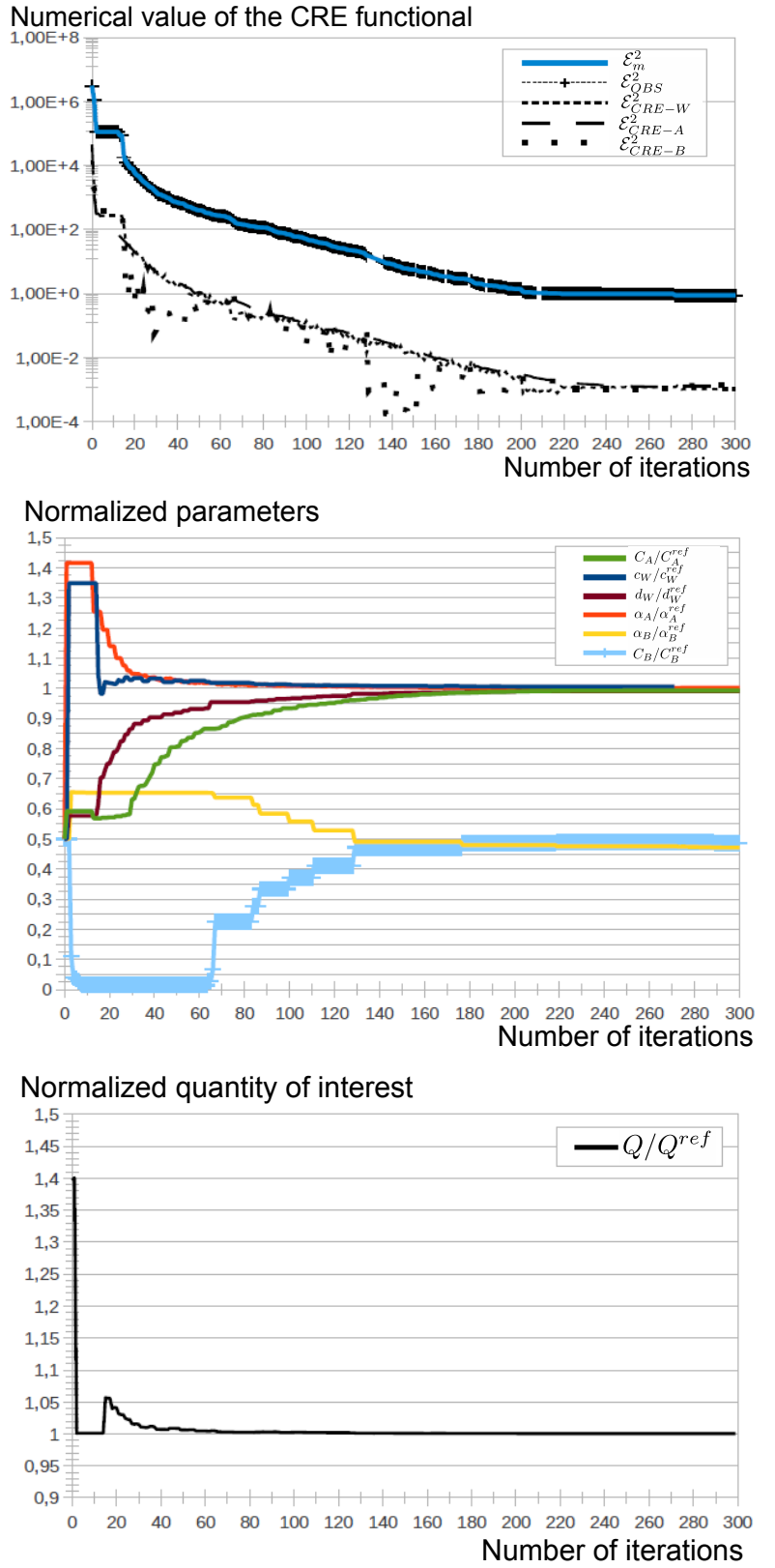

Fig. 10 Modified constitutive relation error, normalized parameters and ratio between the updated quantity of interest and the reference quantity of interest at each iteration - Case of non-corrupted sensor outputs.

between iteration 0 and iteration 3 . In this case, three iterations are sufficient to get an error on the quantity of interest less than 1\% (see Figure 12). If we keep iterating, we see that the exchange coefficient $\alpha_{A}$ between the wall and zone A is exactly determined. The goaloriented being less sensitive to the other parameters, these are not updated. 


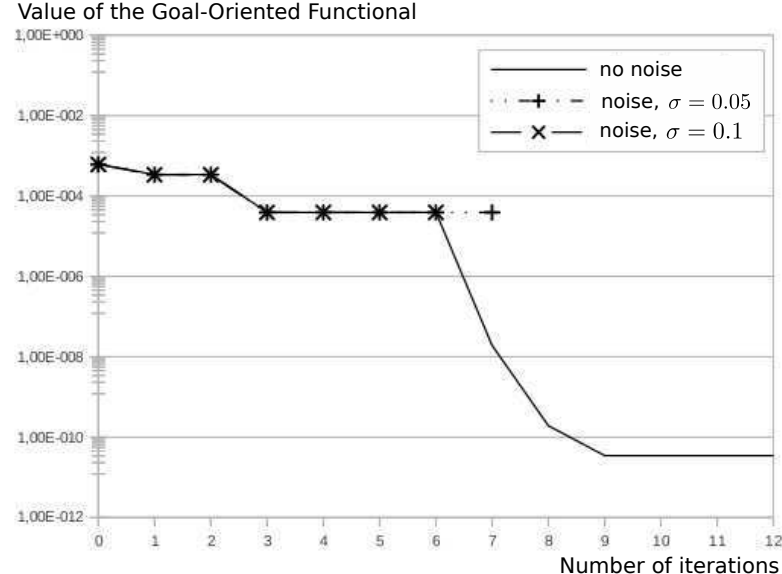

Fig. 11 Goal-oriented functional at each iteration.

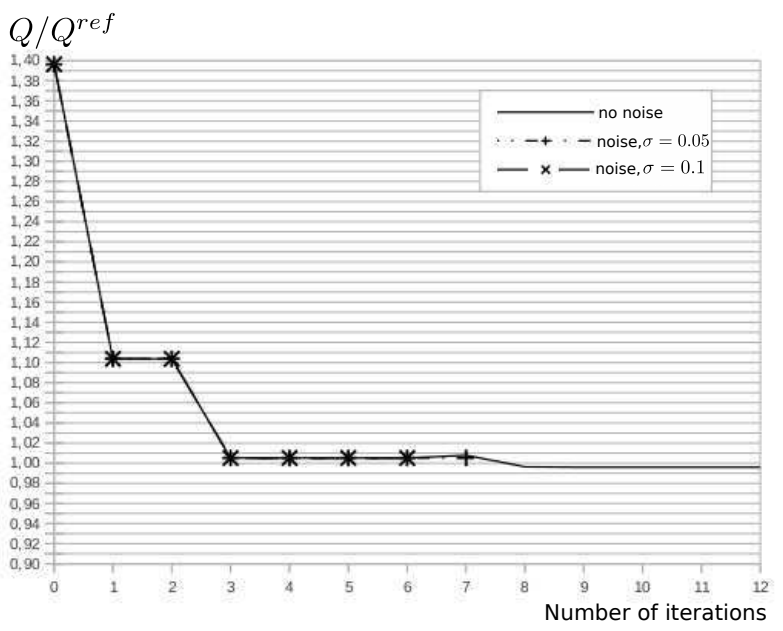

Fig. 12 Ratio of the updated quantity of interest and the reference quantity of interest at each iteration, obtained with the goal-oriented calibration technique.

To study the robustness of the goal-oriented calibration method, the temperature sensor outputs are now corrupted with random errors from a normal distribution $\mathcal{N}\left(0, \sigma^{2}\right)$. The corrupted sensor outputs are represented in Figure 14. Even though it is difficult to distinguish the temporal evolution of the sensor outputs $S_{4}, S_{5}$ and $S_{6}$ when $\sigma=0.1$, the parameters $c_{W}$ and $d_{W}$ are still well-determined by the goal-oriented approach. In Figure 12, we observe that a $1 \%$ error on the quantity of interest can still be obtained after a few iterations when considering corrupted outputs.

At each iteration of the goal-oriented technique, we recall that two unsteady problems and $\mathrm{N}+2$ forward/backward problems have to be solved (see Appendix A). Nevertheless, in this numerical example, 10 iterations are sufficient to update the parameters, so that the goaloriented updating technique remains about 20 times

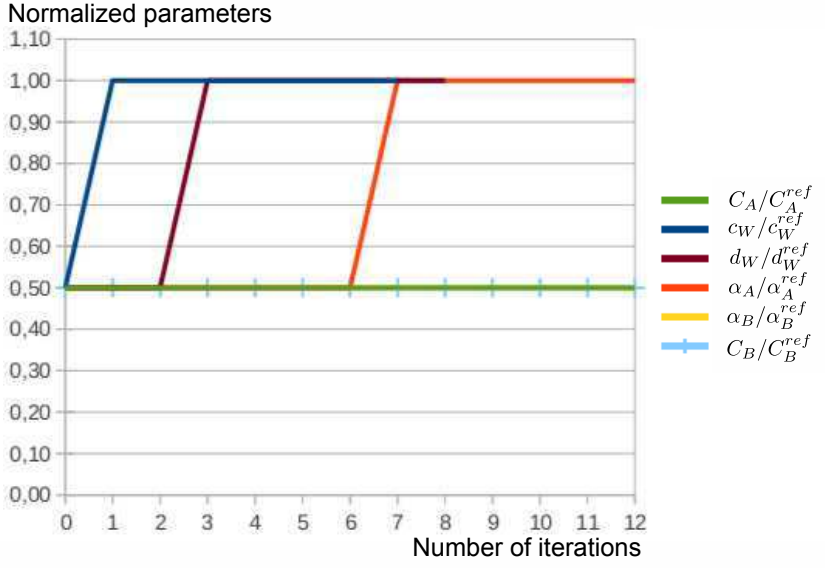

Fig. 13 Updated parameters $\mathbf{p}$ with the goal-oriented calibration technique - Case of non-corrupted sensor outputs.

faster than the classical CRE calibration (the computation time of the unsteady problem is negligible compared to that of the forward/backward coupled problem).
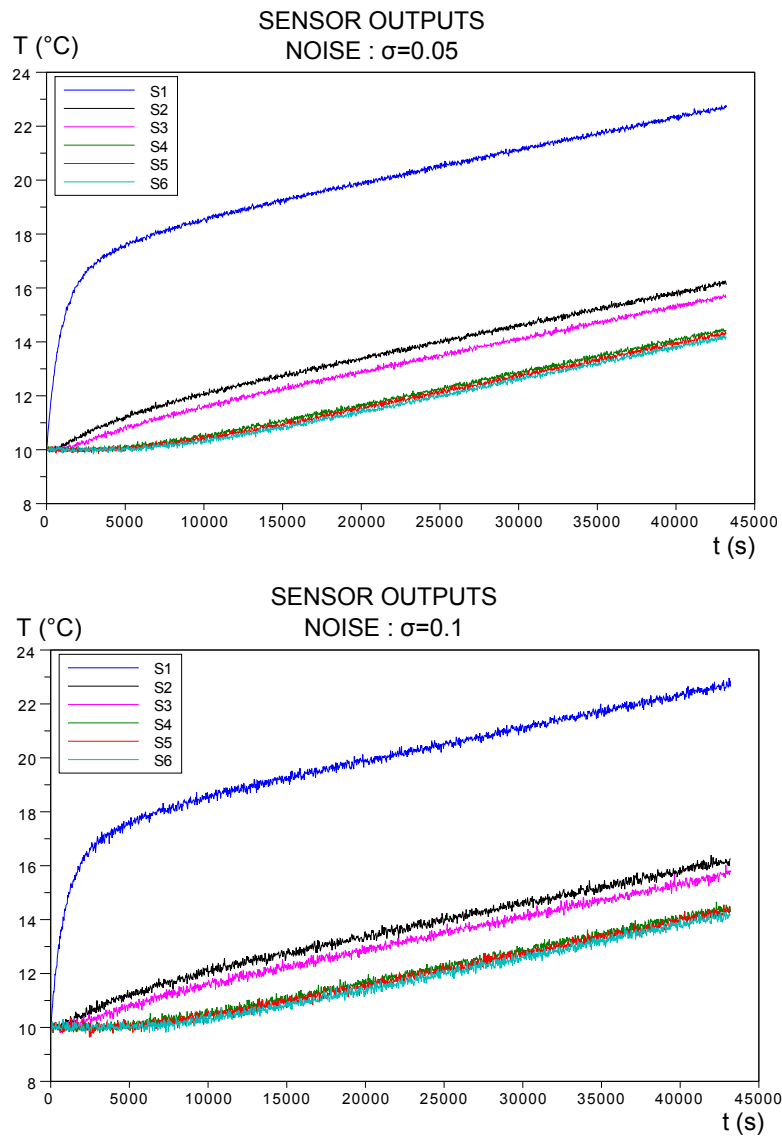

Fig. 14 Corrupted sensor outputs. 
CORRUPTED SENSOR OUTPUTS

$$
\sigma=0.05
$$

\section{Normalized parameters}

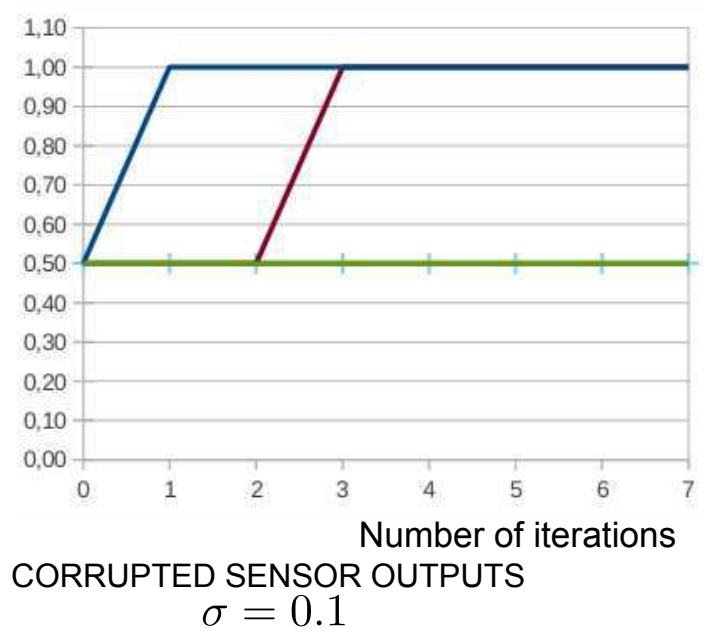

Normalized parameters

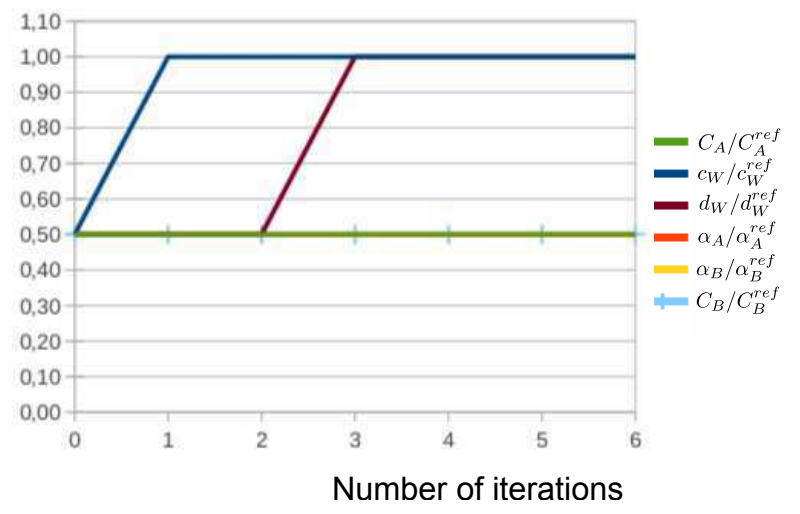

Fig. 15 Parameters p updated with the goal-oriented method - Case of corrupted sensor outputs.

\section{Conclusions and prospects}

We defined and analyzed a new updating strategy dedicated to the prediction of quantities of interest. Based on the modified CRE framework, it uses dedicated cost functions and leads, compared to classical updating methods and with the same amount of experimental data, to a partial updated model in which only relevant parameters with respect to the quantity of interest are corrected. Therefore, the obtained model may be scientifically invalid but remains valid for the objective of the simulation. We focused on linear elasticity models but now wish to extend the method to more complex mechanical models (dynamics, visco-elasticity, plasticity,...) for which the concept of constitutive relation error still applies. Actually, CRE functionals can be formulated for all generalized standard materials in terms of local free energy and dissipation potential to- gether with their Legendre-conjugate dual potentials. Furthermore, the capability to lead sensibility analysis of quantities of interest with respect to measurements opens new research topics related to the optimization of experimental protocoles (sensors location, applied loadings, geometry of the sample); this will be the subject of future works.

\section{A Parameter updating in the unsteady case}

We consider the updating of parameters $\mathbf{p}$ associated with the following semi-discrete equation:

$\widetilde{\mathbb{C}}(\mathbf{p}) \frac{\partial \mathbf{U}}{\partial t}+\widetilde{\mathbb{K}}(\mathbf{p}) \mathbf{U}=\widetilde{\mathbf{F}}(\mathbf{p}), \quad \mathbf{U}(t=0)=\mathbf{T}^{\mathbf{0}}$

Equations (54-56) can be written under this form.

\section{A.1 Classical updating with the constitutive relation} error

For the unsteady problem (59), the discretized modified CRE reads:

$$
\begin{aligned}
\mathcal{E}_{m}^{h 2}(\mathbf{p}, \mathbf{U}, \mathbf{V}) & =\frac{1}{2} \int_{0}^{T}(\mathbf{U}-\mathbf{V})^{T} \mathbb{K}(\mathbf{U}-\mathbf{V}) \mathrm{d} t \\
& +\frac{1}{2} \int_{0}^{T} \frac{r}{1-r}\left(\mathbf{U}-\mathbf{U}_{o b s}\right)^{T} \mathbb{G}_{u}\left(\mathbf{U}-\mathbf{U}_{o b s}\right) \mathrm{d} t
\end{aligned}
$$

where $\mathbf{U}$ is a kinematically admissible temperature which is equal to $\mathbf{T}^{\mathbf{0}}$ at the initial time, and $\mathbf{V}$ is a statically admissible temperature. The pair $(\mathbf{U}, \mathbf{V})$ should satisfy the discretized equilibrium equation:

$\mathbb{C} \frac{\partial \mathbf{U}}{\partial t}+\mathbb{K} \mathbf{V}=\mathbf{F}$

To determine the pair $(\mathbf{U}, \mathbf{V})$ in the localization step, we introduce the Lagrangian:

$L(\mathbf{U}, \mathbf{V}, \boldsymbol{\Lambda})=\mathcal{E}_{m}^{h 2}(\mathbf{p}, \mathbf{U}, \mathbf{V})-\int_{0}^{T} \boldsymbol{\Lambda}^{T}\left(\mathbb{C} \frac{\partial \mathbf{U}}{\partial t}+\mathbb{K} \mathbf{V}-\mathbf{F}\right) \mathrm{d} t$

Finding its stationary point leads to the system:

$$
\begin{aligned}
\widetilde{\mathbb{C}} \frac{\partial \boldsymbol{\Lambda}}{\partial t}+\widetilde{\mathbb{K}}(\mathbf{U}-\mathbf{V})+\frac{r}{1-r} \widetilde{\mathbb{G}}_{u}\left(\mathbf{U}-\mathbf{U}_{o b s}\right) & =\mathbf{0} \\
\mathbb{K}(\mathbf{U}-\mathbf{V})+\mathbb{K} \boldsymbol{\Lambda} & =\mathbf{0} \\
\mathbb{C} \frac{\partial \mathbf{U}}{\partial t}+\mathbb{K} \mathbf{V} & =\mathbf{F} \\
\mathbf{U}(t=0) & =\mathbf{T}^{\mathbf{0}} \\
\boldsymbol{\Lambda}(t=T) & =\mathbf{0}
\end{aligned}
$$

The adjoint solution verifies $\boldsymbol{\Lambda}=\mathbf{V}-\mathbf{U}$ and has null final conditions. From (63), we obtain the following forward/backward coupled system:

$\left[\begin{array}{ll}\mathbb{C} & \mathbb{O} \\ \mathbb{O} & \widetilde{\mathbb{C}}\end{array}\right] \cdot\left(\begin{array}{l}\frac{\partial \mathbf{U}}{\partial t} \\ \frac{\partial \hat{\Lambda}}{\partial t}\end{array}\right)+\left[\begin{array}{cc}\mathbb{K} & \mathbb{K} \\ \frac{r}{1-r} \widetilde{\mathbb{G}}_{u} & -\widetilde{\mathbb{K}}\end{array}\right] \cdot\left(\begin{array}{c}\mathbf{U} \\ \Lambda\end{array}\right)=\left(\begin{array}{c}\mathbf{F} \\ \frac{r}{1-r} \widetilde{\mathbb{G}}_{u} \mathbf{U}_{o b s}\end{array}\right)$ 
with $\mathbf{U}(t=0)=\mathbf{T}^{\mathbf{0}}$ and $\boldsymbol{\Lambda}(t=T)=\mathbf{0}$. A similar system was obtained in elastodynamics model updating using the constitutive relation error [2].

Then, in the correction step, parameters associated to the highest contributions to the modeling error are updated. We underline the fact that, at each iteration of the updating procedure, the coupled system (64) of forward/backward solutions has to be solved $(\mathrm{N}+1)$ times (one solution during the localization step and $\mathrm{N}$ solutions during the correction step).

\section{A.2 Goal-oriented updating with the constitutive} relation error

In the goal-oriented approach, we search for control parameters minimizing the error on the quantity of interest defined by:

$\mathcal{F}_{Q}^{h}(\mathbf{p})=\frac{1}{2} r\left[Q\left(\mathbf{U}_{1}(\mathbf{p})\right)-Q\left(\mathbf{U}_{2}(\mathbf{p})\right)\right]^{2}$

where:

- $Q\left(\mathbf{U}_{1}(\mathbf{p})\right)$ represents the quantity of interest defined from the model only. In this case, $\mathbf{U}_{1}$ verifies the unsteady equation (59);

- $Q\left(\mathbf{U}_{2}(\mathbf{p})\right)$ corresponds to the quantity of interest defined from an interpolation of measurements based on the constitutive relation error. In the unsteady case, the kinematically admissible field $\mathbf{U}_{2}$ and the adjoint field $\boldsymbol{\Lambda}_{2}$ verify the forward-backward system (64). The adjoint field $\boldsymbol{\Lambda}_{2}$ has a null final condition and the kinematically admissible temperature $\mathbf{U}_{2}$ at the initial time is equal to $\mathbf{T}^{\mathbf{0}}$. The statically admissible field can be deduced as $\boldsymbol{\Lambda}_{2}=\mathbf{V}_{2}-\mathbf{U}_{2}$.

We introduce the semi-discrete Lagrangian $L_{Q}$ defined as:

$$
\begin{aligned}
L_{Q}\left(\mathbf{p}, \mathbf{U}_{1}, \mathbf{U}_{2}, \mathbf{V}_{2}, \boldsymbol{\Lambda}_{1}, \boldsymbol{\Lambda}_{2}, \boldsymbol{\Upsilon}_{2}\right)=\frac{1}{2} r\left[Q\left(\mathbf{U}_{1}(\mathbf{p})\right)-Q\left(\mathbf{U}_{2}(\mathbf{p})\right)\right]^{2} \\
-\int_{0}^{T} \boldsymbol{\Lambda}_{1}^{T}\left(\widetilde{\mathbb{C}} \frac{\partial \mathbf{U}_{1}}{\partial t}+\widetilde{\mathbb{K}} \mathbf{U}_{1}-\widetilde{\mathbf{F}}\right) \mathrm{d} t \\
-\int_{0}^{T} \boldsymbol{\Lambda}_{2}^{T}\left(\mathbb{C} \frac{\partial \mathbf{U}_{2}}{\partial t}+\mathbb{K} \mathbf{V}_{2}-\mathbf{F}\right) \mathrm{d} t \\
-\int_{0}^{T} \boldsymbol{\Upsilon}_{2}^{T}\left\{\widetilde{\mathbb{C}}\left(\frac{\partial \mathbf{V}_{2}}{\partial t}-\frac{\partial \mathbf{U}_{2}}{\partial t}\right)+\frac{r}{1-r} \widetilde{\mathbb{G}}_{u} \mathbf{U}_{2}\right. \\
\left.-\widetilde{\mathbb{K}}\left(\mathbf{V}_{2}-\mathbf{U}_{2}\right)-\frac{r}{1-r} \widetilde{\mathbb{G}}_{u} \mathbf{U}_{o b s}\right\} \mathrm{d} t
\end{aligned}
$$

To find the saddle-point of $L_{Q}, \mathbf{U}_{1}$ (resp. $\left(\mathbf{U}_{2}, \mathbf{V}_{2}\right)$ ) has to satisfy (59) (resp. (64)), $\boldsymbol{\Lambda}_{1}$ should verify the additional adjoint equation:

$\widehat{\mathbb{C}} \frac{\partial \boldsymbol{\Lambda}_{1}}{\partial t}-\widehat{\mathbb{K}} \boldsymbol{\Lambda}_{1}=-\mathbf{B}_{Q}, \quad \boldsymbol{\Lambda}_{1}(t=T)=\mathbf{0}$

and $\left(\boldsymbol{\Lambda}_{2}, \boldsymbol{\Upsilon}_{2}\right)$ should verify the additional coupled forward/backward system:

$$
\left[\begin{array}{ll}
\widetilde{\mathbb{C}} & -\widehat{\mathbb{C}} \\
\mathbb{O} & \widetilde{\mathbb{C}}
\end{array}\right] \cdot\left(\begin{array}{l}
\frac{\partial \boldsymbol{\Lambda}_{2}}{\partial t} \\
\frac{\partial \boldsymbol{\Upsilon}_{2}}{\partial t}
\end{array}\right)+\left[\begin{array}{cc}
\mathbb{O} & -\frac{r}{1-r} \widehat{\mathbb{G}}_{u}-\widehat{\mathbb{K}} \\
-\mathbb{K} & \widetilde{\mathbb{K}}
\end{array}\right] \cdot\left(\begin{array}{l}
\boldsymbol{\Lambda}_{2} \\
\boldsymbol{\Upsilon}_{2}
\end{array}\right)=\left(\begin{array}{c}
\mathbf{B}_{Q} \\
\mathbf{0}
\end{array}\right)
$$

with $\boldsymbol{\Lambda}_{2}(t=0)=\mathbf{0}$ and $\boldsymbol{\Upsilon}_{2}(t=T)=\mathbf{0}$. Square matrices $\widehat{\mathbb{C}}, \widehat{\mathbb{K}}$ and $\widehat{\mathbb{G}}_{u}$ are obtained from $\mathbb{C}, \mathbb{K}$ and $\mathbb{G}_{u}$ by removing lines and columns corresponding to prescribed dofs for $\mathbf{U}_{1}$ and $\mathbf{U}_{2}$. The vector of nodal forces $\mathbf{B}_{Q}$ is associated to the term $\beta Q^{\prime}(\mathbf{U} ; \delta \mathbf{U})$ with $\beta=r\left[Q\left(\mathbf{U}_{1}(\mathbf{p})\right)-Q\left(\mathbf{U}_{2}(\mathbf{p})\right)\right]$.

In order to evaluate the gradient of $\mathcal{F}_{Q}^{h}$, the solutions of two unsteady problems (59) and (67), and two forward/backward problems (64) and (68) are required. Then, as in the steady case (see Section 4.1), we update parameters associated with high components to the gradient of $\mathcal{F}_{Q}^{h}$. During this minimization step, $\mathrm{N}$ solutions of the forward/backward coupled problem are required. To summarize, at each iteration of the goal-oriented approach, two unsteady problems and $(\mathrm{N}+2)$ forward/backward coupled problems have to be solved. It is more expensive than one iteration of CRE-based calibration. Nevertheless, we observe in the thermal building application presented in Section 6.3 that the number of iterations is about twenty times smaller in the goal-oriented calibration technique.

\section{References}

1. O. Allix, P. Feissel, H.M. Nguyen. Identification strategy in the presence of corrupted measurements. Engineering Computations, 2005; 22(5-6):487-504.

2. P. Feissel, O. Allix. Modified constitutive relation error identification strategy for transient dynamics with corrupted data : the elastic case. Computer Methods in Applied Mechanics and Engineering, 2007; 196(1316):1968-1983.

3. S. Andrieux, H.D. Bui. Ecart à la réciprocité et identification de fissures en thermoélasticité isotrope transitoire. Comptes Rendus Mécanique, 2006; 334(4):225-229.

4. S.R. Arridge. Optical tomography in medical imaging. Inverse Problems, 1999; 15:R41.

5. S. Avril, M. Bonnet, A-S. Bretelle, M. Grédiac, F. Hild, P. Ienny, F. Latourte, D. Lemosse, S. Pagano, E. Pagnacco, F. Pierron. Overview of identification methods of mechanical parameters based on full-field measurements. Experimental Mechanics, 2008; 48:381-402.

6. R. Becker, B. Vexler. A posteriori error estimation for finite element discretization of parameter identification problems. Numerische Mathematik, 2004; 96:435-459.

7. M. Bonnet, J. Ben Abdallah. Structural parameter identification using nonlinear gaussian inversion. Inverse Problems in Engineering Mechanics, 1994; 235-242.

8. M. Bonnet, A. Constantinescu. Inverse problems in elasticity. Inverse Problems, 2005; 21:R1-R50.

9. S. Bonettini, F. Benvenuto, R. Zanella, L. Zanni, M. Bertero. Gradient projection approaches for optimization problems in image debluring and denoising. in Proceedings of the 17th European Signal Processing Conference, $2009 ; 1384-1388$.

10. R. Bouclier, F. Louf, L. Chamoin. Real-time validation of mechanical models coupling PGD and constitutive relation error. Computational Mechanics, 2013; 52(4):861883.

11. H.D. Bui, A. Constantinescu. Spatial localization of the error of constitutive law for the identification of defects in elastic bodies. Arch. Mech., 2000; 52:511-522. 
12. G. Cailletaud, P. Pilvin. Identification and inverse problems related to material behaviour. In H.D. Bui, M. Tanaka, and al., editors, Inverse Problems in Engineering Mechanics, 1994; 79-86.

13. A. Chouaki, P. Ladevèze, L. Proslier. An updating of structural dynamic model with damping. Inverse Problems in Engineering : Theory and Practice, 1996; 335342 .

14. A. Deraemaeker, P. Ladevèze, P. Leconte. Reduced bases for model updating in structural dynamics based on constitutive relation error. Computer Methods in Applied Mechanics and Engineering, 2002; 191:2427-2444.

15. A. Deraemaeker, P. Ladevèze, T. Romeuf. Model validation in the presence of uncertain experimental data. Engineering Computations, 2004; 21(8):808-833.

16. O. Dorn. Scattering and absorption transport sensitivity functions for optical tomography. Optics Express, 2000; 7(13):492-506.

17. B. Faverjon, P. Ladevèze, F. Louf. Validation of stochastic linear structural dynamics models. Computers \& Structures, 2009; 87(13-14):829-837.

18. P. Feissel, O. Allix. Modified constitutive relation error identification strategy for transient dynamics with corrupted data : the elastic case. Computer Methods in Applied Mechanics and Engineering, 2007; 196(1316):1968-1983.

19. F. Gant, P. Rouch, F. Louf, L. Champaney. Definition and updating of simplified models of joint stiffness. International Journal of Solids \& Structures, 2011; 48(5):775784 .

20. B. Halphen, Q.S. Nguyen. Sur les matériaux standards généralisés. Journal de Mécanique, 2006; 14(1):39-63.

21. P.C. Hansen. Rank-deficient and discrete ill-posed problems. SIAM, Philadelphia, 1998.

22. F. Hild, S. Roux. Digital image correlation: from measurement to identification of elastic properties - a review. Strain, 2006; 42:69-80.

23. T.Z. Hong, Y. Jiang. A new multizone model for the simulation of building thermal performance. Building 86 Environment; 1997; 32(2):123-128.

24. H. Johansson, K. Runesson, F. Larsson. Parameter identification with sensitivity assessment and error computation. GAMM-Mitt, 2007; 2:430-457.

25. H. Johansson, F. Larsson, K. Runesson. Applicationspecific error control for parameter identification problems. International Journal for Numerical Methods in Biomedical Engineering, 2011; 27:608-618.

26. P. Ladevèze, D. Nedjar, M. Reynier. Updating of finite element models using vibration tests. AIAA Journal, 1994; 32(7):1485-1491.

27. P. Ladevèze, A. Chouaki. Application of a posteriori error estimation for structural model updating. Inverse Problems, 1999; 15(1):49-58.

28. P. Ladevèze, J-P. Pelle. Mastering Calculations in Linear and Nonlinear Mechanics. Springer NY, 2004.

29. P. Ladevèze, G. Puel, A. Deraemaeker, T. Romeuf. Validation of structural dynamics models containing uncertainties. Computer Methods in Applied Mechanics and Engineering, 2006; 195(4-6):373-393.

30. A. Nassiopoulos, F. Bourquin. On-site building walls characterization. Numerical Heat Transfer, Part A: Application: An International Journal of Computation and Methodology, 2013; 63(3):179-200.
31. J.A. Nelder, R. Mead. A simplex method for function minimization. Computer Journal, 1965; 308-313.

32. W. Oberkampf, T. Trucano, C. Hirsh. Verification, Validation and Predictive Capability in Computational Engineering and Physics. Technical report, Sandia 2003-3769, 2003.

33. K. Popper. The Logic of Scientific Discovery. Routledge Classics, Taylor and Francis, 2003.

34. P.J. Roache. Verification and Validation in Computational Science and Engineering. Hermosapublishers, 1998.

35. B.V. Rosic, A. Kucerova, J. Sykora, O. Pajonk, A. Litvinenko, H. Matthies. Parameter identification in a probabilistic setting. Engineering Structures, 2013; 50:179196.

36. M.A. Sutton, J.J. Orteu, H. Schreier. Image correlation for shape, motion and deformation measurements: basic concepts, theory and applications. Springer, 2009.

37. A. Tarantola. Inverse problem theory and model parameter estimation. SIAM, 2005.

38. A.N. Tikhonov, Y. Arsenin. Solutions to ill-posed problems. Wintson-Widley, New York, 1977. 